\title{
Long non-coding RNA H19 contributes to wound healing of diabetic foot ulcer
}

\author{
Bo Li ${ }^{1, *}$, Yue Zhou ${ }^{2, *}$, Jing Chen², Tingting Wang2 ${ }^{2}$ Zhijuan $\mathrm{Li}^{2}$, Yili Fư ${ }^{3}$, Aixia Zhai ${ }^{4}$ and Changlong $\mathrm{Bi}^{5}$ \\ 1Department of Endocrinology, The Fourth Hospital of Harbin Medical University, Harbin, People's Republic of China \\ ${ }^{2}$ Comprehensive Second Department, The Fourth Affiliated Hospital of Harbin Medical University, Harbin, People's Republic of China \\ ${ }^{3}$ School of Life Science and Technology, Harbin Institute of Technology, Harbin, People's Republic of China \\ ${ }^{4}$ Department of Laboratory Medicine, The Eighth Affiliated Hospital, Sun Yat-sen University, Shenzhen, People's Republic of China \\ ${ }^{5}$ Department of Endocrinology, The Eighth Affiliated Hospital, Sun Yat-sen University, Shenzhen, People's Republic of China
}

Correspondence should be addressed to A Zhai or C Bi: aixiazhai@126.com or bichanglongdr@163.com

*(B Li and Y Zhou contributed equally to this work)

\begin{abstract}
Diabetic foot ulcer (DFU) is a chronic and non-healing complication of diabetes that leads to high hospital costs and, in extreme cases, to amputation. Recent studies have reported that long non-coding RNAs (IncRNAs) are linked to various diabetes-related symptoms. Thus, we aim to explore the role of IncRNA H19 in the wound healing process following DFU. Fibroblasts were isolated from the ulcer margin tissues of DFU patients, with the expression of IncRNA H19, connective tissue growth factor (CTGF) or serum response factor (SRF) altered by lentivirus infection. Next, rat models of DFU induced by high glucose and lipid diet were established, which was also infected with the corresponding lentivirus. The interaction among InCRNA H19, SRF and CTGF was determined. Afterward, cell proliferation and apoptosis, angiogenesis, ECM remodeling and wound healing in DFU tissues were evaluated to explore the effects of InCRNA H19/SRF/CTGF and MAPK signaling pathway on DFU. CTGF was poorly expressed in ulcer tissues from DFU rats and patients. CTGF overexpression was shown to activate the MAPK signaling pathway to promote cell proliferation, ECM remodeling, angiogenesis and wound healing while inhibiting cell apoptosis. IncRNA H19 was validated to elevate CTGF expression by recruiting SRF to the promoter region of CTGF, thus accelerating cell proliferation, ECM remodeling and wound healing while repressing cell apoptosis. Furthermore, MAPK signaling pathway activation is confirmed to be the underlying mechanism behind IncRNA H19/CTGF/SRF-induced results. Thus, IncRNA H19 accelerated wound healing in DFU through elevation of CTGF and activation of the MAPK signaling pathway.
\end{abstract}

\section{Key Words}

- diabetic foot ulcer

- long non-coding RNA H19

- connective tissue growth factor

- serum response factor

- MAPK signaling pathway

- wound healing

\section{Introduction}

Diabetic foot ulcer (DFU) is a common complication of diabetes mellitus and a major cause of morbidity and hospitalization in DF patients, which induces infection, gangrene, amputation and even death (Yazdanpanah et al. 2015). About $15 \%$ of patients with diabetes develop
DFU, who usually suffer from wound ulceration caused by loss of wound healing or even pain of amputation (Goodson 2016). DFU is primarily induced by repetitive pressure over a region that endures heavy shear or vertical pressure in peripheral neuropathy patients 
(Armstrong et al. 2017). Risk factors for DFU include infection, cigarette smoking, absence of training, diagnosis of retinopathy, duration of diabetes, advanced age, HbA1c and diabetic neuropathy (Turns 2012, Shahbazian et al. 2013). Currently, preventative measures to stave off disease deterioration involve improved glycaemia control and wound protection (Turns 2012). Many treatments for DFU have been developed, which include antibiotics usage, a variety of wound protective dressings that also promote wound healing (Dumville et al. 2012), hyperbaric oxygen therapy (Kranke et al. 2015), and stem cell therapy (Blumberg et al. 2012).

The critical link in treatment is to understand the pathophysiology of DFU, which comprises formation of extracellular matrix and altered metabolism (Loots et al. 1998, Goldin et al. 2006). Many macromolecules have been shown to participate in these physiological events. Serum response factor (SRF), a transcription factor for regulating growth and cytoskeletal pathway, is correlated with the pathogenesis of type 2 diabetes (Jin et al. 2011). Connective tissue growth factor (CTGF) is a matricellular protein from the Cyr61/CTGF/Nov (CCN) protein family, which interacts with extracellular matrix (ECM) proteins to mediate external signal transduction into cells through many subtypes of integrin receptors ( $\mathrm{Pi}$ et al. 2015). CTGF has been newly identified as a predictor for further procession of cardiovascular death and myocardial infarction in patients with type 2 diabetes (Hunt et al. 2018). According to a recent study by Henshaw et al., CTGF plays a functional role in the treatment of DFU (Henshaw et al. 2015).

Long non-coding RNAs (lncRNAs) have been shown to be involved in transcriptional regulation in multiple diseases (Hon et al. 2017). For instance, lncRNAs are abnormally expressed in early diabetic retinopathy and are associated with the pathogenesis of diabetic retinopathy by regulating various pathogenetic pathways (Yan et al. 2014). Notably, lncRNA H19 has been identified as an upstream modulator of gluconeogenesis and a regulator of hepatic glucose metabolism, and its lower hepatic level has been detected in diabetic mice (Goyal et al. 2019). IncRNA H19 has been demonstrated to be a promising useful biomarker and predictive target for the diagnosis of type 2 diabetes in colorectal cancer patients (Zhao et al. 2019). Additionally, downregulated early growth response 1 (EGR1) expression leads to inhibited lncRNA H19 expression, thus forming a negative feedback loop that is essential for maintaining the homeostasis of vitamin D receptor (VDR) and reducing the incidence of diabetic nephropathy (Fan et al. 2019). IncRNAs have also been shown to function as a mediator of the mitogenactivated protein kinase (MAPK) signaling pathway in cancer (Tasharrofi \& Ghafouri-Fard 2018). Activated MAPK signaling pathway is considered as a regulator of the inflammatory response, suggesting the potential role of the MAPK signaling pathway in treating autoimmune and inflammatory diseases (Arthur \& Ley 2013). Besides, MAPK transduces various signaling pathways and is associated with obesity and diabetes in response to multiple ligands and cell stimuli (Lawrence et al. 2008). Based on the aforementioned studies, we hypothesized that lncRNA H19 affects wound healing in DFU through regulating SRF, CTGF and MAPK signaling pathway. To test this hypothesis, we developed a rat model of DFU induced by streptozotocin (STZ) and then conducted in vivo and in vitro experiments in order to offer a deep understanding for the underlying molecular mechanisms in DFU progression, thus providing possible therapeutic strategies for DFU patients.

\section{Materials and methods}

\section{Ethics statement}

The study was approved by the Ethics Committee of the Fourth Hospital of Harbin Medical University and conducted in strict accordance to the Declaration of Helsinki. All participants signed written informed consents prior to the study. The protocol of animal experiments was also approved by the Institutional Animal Care and Use Committee of The Fourth Hospital of Harbin Medical University. Animal experiments were performed in strict accordance with the recommendations in the Guide for the Care and Use of Laboratory Animals published by the US National Institutes of Health. All efforts were made to minimize the number and suffering of the included animals.

\section{Study subjects}

A total of 25 foot ulcer tissues were collected from DFU patients (aged 42-77 years, with a mean age of $64.20 \pm 7.26$ years) who underwent surgery at The Fourth Hospital of Harbin Medical University. According to the Wagner grade for DFU, patients graded from level 1 to 3 did not have serious lower extremity venous disease, serious cardiovascular and cerebrovascular disease and other severe complications (male:female ratio, 3:2) (Wagner 1981). At the same time, a total of 20 cases of foot ulcer 
tissues were collected from patients without a history of DF as controls.

\section{Isolation and culture of fibroblasts}

The fibroblasts were isolated from the ulcer margin of DFU patients. Briefly, the tissue blocks were collected from the ulcer margin of DFU patients and then placed in sterile Dulbecco's modified Eagle's medium (DMEM) supplemented with 10\% fetal bovine serum (FBS) (Gibco). Next, the tissue blocks were washed with PBS containing $100 \mathrm{U} / \mathrm{mL}$ penicillin and streptomycin (Gibco) for two to three times. After s.c. adipose tissues were stripped, the tissue blocks were cut into $0.5-1 \mathrm{~mm}^{3}$ slices. Next, the tissue slices were uniformly covered in a $25-\mathrm{cm}$ culture bottle, added with 2-3 mL DMEM containing 10\% FBS and then incubated at $37^{\circ} \mathrm{C}$ with $95 \%$ air and $5 \% \mathrm{CO}_{2}$ for $4 \mathrm{~h}$. Cellular morphology was then observed, photographed and recorded. Upon reaching $80-90 \%$ confluence, the cells were passaged in separate culture bottles. Cells at passage 3-5 were collected for further use. Subsequently, fibroblasts from the ulcer margin of DFU patients were seeded and cultured on sterile coverslips in 24-well plates, then fixed in $4 \%$ paraformaldehyde solution for $10 \mathrm{~min}$, and treated with PBS containing 0.1\% Triton X-100 on ice for $10 \mathrm{~min}$ of punching and film breaking. Next, the cells were immersed in $3 \% \mathrm{H}_{2} \mathrm{O}_{2}$ for 10 min to eliminate endogenous peroxisome and blocked with 10\% goat serum for $30 \mathrm{~min}$. The cells were subsequently probed with primary antibody to mouse anti-human vimentin (ab3974, 1:20) or keratin (ab8068, 1:1000) at $4^{\circ} \mathrm{C}$ for $3 \mathrm{~h}$. Following three rinses with PBS (10 min/time), the cells were reprobed with the secondary antibody of goat antimouse (ab6789, 1:1000) in a $37^{\circ} \mathrm{C}$ wet box for $30 \mathrm{~min}$, followed by PBS washing for three times ( $10 \mathrm{~min} /$ time). The antibodies were all purchased from Abcam. Finally, the cells were developed with diaminobenzidine (DAB), counterstained with hematoxylin (nucleus), differentiated using $1 \%$ hydrochloric acid ethanol, turned blue with diluted ammonia, dehydrated with gradient ethanol and xylene, and mounted using neutral resin.

\section{DFU rat model establishment and treatment}

A total of 104 Sprague-Dawley male rats were purchased from the experimental animal center of Heilongjiang University of Traditional Chinese Medicine (Heilongjiang, China) (aged 6-8 weeks; weighing 171.06-242.36 g). The rats were housed in individual cages in a $12 \mathrm{~h}$ light: $12 \mathrm{~h}$ darkness cycle with free access to food and water.
After adaptive feeding for 1 week, eight rats were fed with common diet as normal controls, while the remaining 96 rats were fed with high glucose and high lipid diet. After 6 weeks, these rats were injected with STZ (S0130-50MG, Sigma-Aldrich) to develop DFU models. The rats following successful model construction presented many symptoms, such as more food, polydipsia, polyuria, weight loss, and blood glucose increase $(16.7 \mathrm{mmol} / \mathrm{L})$. The model success rate was $83.72 \%$, with 80 rats successfully modeled. Then the successfully modeled 80 rats were divided into 10 groups, with 8 rats in each group. Then, $0.45 \%$ STZ diluted by $0.1 \mathrm{mmol} / \mathrm{L}$ sterile sodium citrate buffer solution (45 mg/kg) was injected intraperitoneally to the rats. After $72 \mathrm{~h}$, the off-line blood glucose monitoring system Glucotrend 2 (RocheDiagnostics) was used to determine the blood glucose level from the tip of the tail vein. Rats with a stable blood glucose level of $\geq 16.7 \mathrm{mmol} / \mathrm{L}$ for 10 days were enrolled in the following experiment (Ye et al. 2017).

A foot ulcer model was generated in STZ-induced diabetic mice as previously described (Gan et al. 2019). Briefly, after the DFU rat model was established for 2 weeks, the rats were anesthetized with 3\% sodium pentobarbital (P3761, Sigma-Aldrich) which was injected near the wound surface. Then, the hair on the right side of the foot was shaved after disinfection and the remaining hair was removed with a depilatory agent. A mark ( $6 \mathrm{~mm} \times$ $6 \mathrm{~mm}$ ) was created on foot of rat with a sterile perforator, and the whole skin of the mark was excised. The rats' wounds were transduced with various lentiviral vectors $\left(1 \times 10^{9} \mathrm{pfu} / 100 \mu \mathrm{L}\right)$ and treated with $10 \mu \mathrm{M}$ DMSOdiluted MAPK signaling pathway inhibitor. Specifically, near the wound surface, DFU rats were injected with lentiviruses of overexpression negative control (oe-NC), oe-CTGF, oe-CTGF+MAPK inhibitor, oe-H19, shRNA-NC, sh-SRF, oe-H19+sh-NC, oe-H19+sh-SRF and oe-H19+sh-CTGF ( $n=8$ in each group). After $0,3,7,14$ and 21 days of injection, blood glucose was determined and body weight and average water intake were recorded. At last, 21 days after the formation of DFU, both lesions and adjacent skin of the foot dorsum were collected, fixed with $10 \%$ paraformaldehyde solution and then embedded in paraffin for histological staining and cell apoptosis determination.

\section{Construction and transfection of lentiviral vectors}

PCR was utilized to amplify target genes. Next, the amplified products were cloned into the lentiviral overexpression vector pLV-EGFP-N between ECOR1 and NOT1 using a Cold Fusion kit and shRNAs were then 
inserted into the lentivirus shRNA fluorescence-expressed gene silencing vector pSIH1-H1-copGFP. Then, the shRNA sequences were synthesized by Shanghai GenePharma (Shanghai, China). Subsequently, lentiviruses, including pLV-EGFP-H19 (oe-H19), pLV-EGFP-CTGF (oe-CTGF), pSIH1-H1-copGFP-sh-CTGF (sh-CTGF), pSIH1-H1copGFP-sh-SRF (sh-SRF), pSIH1-H1-copGFP-sh-NC (sh-NC) and pLV-EGFP-NC (oe-NC), were packaged in 293T cells which were cultured in DMEM complete medium containing 10\% FBS and then passaged every other day.

Next, fibroblasts the ulcer margin of DFU patients in logarithmic growth phase were trypsinized, made into cell suspension $\left(5 \times 10^{4}\right.$ cells $\left./ \mathrm{mL}\right)$ and then inoculated in a six-well plate for overnight culture at $37^{\circ} \mathrm{C}$. Cells were then infected with 293T cells-packaged lentiviruses $\left(1 \times 10^{8} \mathrm{TU} / \mathrm{mL}\right)$ for $48 \mathrm{~h}$, after which green fluorescent protein (GFP) expression was observed under a fluorescence microscope. In brief, fibroblasts were transfected with sh-NC, sh-CTGF, oe-NC, oe-CTGF, oe-H19, sh-SRF, oe-H19+sh-SRF and oe-H19+sh-CTGF.

\section{Reverse transcription quantitative polymerase chain reaction (RT-qPCR)}

Total RNA was extracted using TRIzol (Invitrogen.) and all primers were synthesized by Invitrogen (Table 1). The extracted RNA was reversely transcribed into cDNA using High-Capacity cDNA Reverse Transcription kit (4368813, Thermo Fisher Scientific). Glyceraldehyde-3-phosphate dehydrogenase (GAPDH) (Invitrogen) was regarded as an internal reference. RT-qPCR experiments were conducted on an ABI 7500 quantitative PCR instrument (Thermo Fisher Scientific) using SYBR®Premix Ex Taq ${ }^{\text {TM }}$ (Tli RNaseH Plus) kit (RR820A, Takara). The PCR reaction was run on a real-time fluorescent quantitative PCR instrument

Table 1 Primer sequences for RT-qPCR.

\begin{tabular}{|c|c|}
\hline Gene & Primer sequences \\
\hline \multirow[t]{2}{*}{ CTGF (human) } & F: 5'-AGTGGACAGAACAGGGCAAA-3' \\
\hline & R: 5'-TCTTCGCACCACTCCTGATT-3' \\
\hline \multirow[t]{2}{*}{ CTGF (rat) } & F: 5'-TGTGCACTGCCAAAGATGGT-3' \\
\hline & R: 5'-GGTACACGGACCCACCGA-3' \\
\hline \multirow[t]{2}{*}{ IncRNA H19 (human) } & F: 5'-TTCCAGGCAGAAAGAGCAAGAGGGC-3' \\
\hline & R: 5'-AGACGTCCTGCTGCAACTCCCCGAG-3' \\
\hline \multirow[t]{2}{*}{ IncRNA H19 (rat) } & F: 5'-CTAGGCTGGGGTCAAACAGG-3' \\
\hline & R: 5'-AGAGCC ACTCTTGAACCTGC-3' \\
\hline \multirow[t]{2}{*}{ GAPDH (rat) } & F: 5'-CTCTCTGCTCCTCCCTGTTC-3' \\
\hline & R: 5'-CACCGACCTTCACCATCTTG-3' \\
\hline \multirow[t]{2}{*}{ GAPDH (human) } & F: 5'-CCATGTTCGTCATGGGTGTGAACCA-3' \\
\hline & R: 5'-GCCAGTAGAGGCAGGGATGATGTTC-3' \\
\hline
\end{tabular}

CTGF, connective tissue growth factor; F, forward; GAPDH, glyceraldehyde3-phosphate dehydrogenase; R, reverse; RT-qPCR, RT quantitative PCR.
(ABI, Oyster Bay, NY, USA). The final data were analyzed using $2^{-\Delta \Delta \mathrm{Ct}}$ method.

\section{Western blot analysis}

Total protein was extracted from tissues or cells with radioimmunoprecipitation assay (RIPA) lysis buffer containing PMSF and protease inhibitor in strict accordance with the instructions. The supernatant was then extracted for determining protein concentration using a bicinchoninic acid (BCA) kit (23227, Thermo Fisher Scientific). After separation by PAGE, the protein was transferred onto a PVDF membrane and then blocked with 5\% BSA at room temperature for $1 \mathrm{~h}$. Subsequently, the membrane was probed overnight at $4^{\circ} \mathrm{C}$ with primary antibodies (Abcam): CTGF (ab6992, 1:1000), ERK (ab196883, 1:1500), p38 (ab170099, 1:1000), Jun N-terminal kinase (JNK) (ab99380, 1:2500), phosphorylated (p)-ERK (ab214362, 1:1000), p-p38(ab47363, 1:1000), p-JNK (ab47337, 1:500), matrix metalloproteinase (MMP)-3 (ab53015, 1:1000), MMP-13 (ab39012, 1:3000), collagen II (ab34712, 1:5000), aggrecan (ab3773, 1:100), collagen I (ab90395, 1:1000), vascular endothelial growth factor (VEGF) (ab46154, $1: 1000$ ), transforming growth factor (TGF)- $\beta 1$ (ab64715, $1: 500), \alpha$-smooth muscle actin ( $\alpha$-SMA) (ab5694, 1:2000) and $\beta$-actin (ab8226, 1:5000). The following day, the membrane was washed with PBS with Tween 20 (PBST) three times (10 $\mathrm{min} /$ time) and then reprobed with secondary antibody horseradish peroxidase (HRP)-labeled goat anti-mouse immunoglobulin G (IgG) (ab6789, 1:5000, Abcam) at room temperature for $1.5 \mathrm{~h}$. Following three washes with PBST (15 min/time), the membrane was visualized with developing solution (NCI4106, Pierce). Image $1.48 \mathrm{u}$ software was used to conduct protein quantitative analysis (Bio-Rad).

\section{Fluorescent in situ hybridization (FISH) assay}

Cells were seeded on a coverslip in a 24 -well plate at a density of $6 \times 10^{4}$ cells/well. When cell confluence reached around $80 \%$, the coverslip was taken out and fixed with $1 \mathrm{~mL} 4 \%$ paraformaldehyde solution at room temperature. After treatment with protease $\mathrm{K}(2 \mu \mathrm{g} / \mathrm{mL})$, glycine and B phthalein reagent, the cells were incubated with $250 \mu \mathrm{L}$ pre-hybridization solution at $42^{\circ} \mathrm{C}$ for $1 \mathrm{~h}$ and then hybridized with $250 \mu \mathrm{L}$ hybridization solution containing $\mathrm{H} 19$ probe $(300 \mathrm{ng} / \mathrm{mL})$ at $42^{\circ} \mathrm{C}$ overnight. Subsequently, the cell nucleus was stained with PBST-diluted DAPI (1:800) dye solution for $5 \mathrm{~min}$, and the coverslip was blocked 
with anti-fluorescence quenching agent. Five visual fields were randomly selected, then observed and photographed under a fluorescence microscope (Olympus).

\section{RNA binding protein immunoprecipitation (RIP) assay}

Cells were lysed using RIPA cell lysis buffer according to the instructions of the RIP kit (17-700, Merck Millipore). In brief, the magnetic beads for immunoprecipitation were added with rabbit anti-human SRF (ab53147, 1:1000, Abcam) to generate the magnetic bead-SRF antibody complex, which was then mixed with cell lysate. After immunoprecipitation, magnetic-bead-SRF antibody-H19 complexes were obtained, which later underwent RNA purification for RT-qPCR.

\section{Chromatin immunoprecipitation (ChIP)-PCR}

Cells were treated with $4 \%$ formaldehyde (final concentration of $1 \%$ ) solution and then sonicated. Next, NC rabbit antibodies (Cell Signaling Technologies) IgG (\#3900) and SRF (\#5147) were added to the cells and combined with the promoter of SRF-CTGF. Then protein A agarose/salmon sperm DNA was added to the cells to combine and precipitate SRF antibody-SRF-CTGF promoter compound. The precipitated compound was subsequently washed to remove non-specific binding and then eluted, after which enriched SRF-CTGF promoter compound was obtained. After decrosslinking, the enriched CTGF promoter fragment was purified for PCR analysis.

\section{Dual-luciferase reporter gene assay}

CTGF promoter sequence and a complete sequence of genes were obtained from National Center of Biotechnology Information (NCBI) database (http://www.ncbi.nlm. nih.gov/gene). CTGF promoter region was cloned into pmirGLO luciferase vector (Promega) to construct pmirGLO-CTGF prom wild type (WT) vector. SRF siRNA (si-SRF) was purchased from Shanghai GenePharma. Next, according to the instructions of lipo2000, cells were treated with CTGF prom WT+pLV-EGFP-control, CTGF prom $\mathrm{WT}+$ si-NC, CTGF prom $\mathrm{WT}+$ si-SRF, CTGF prom $\mathrm{WT}+$ pLV-EGFP-N-H19, CTGF prom WT + pLV-EGFPN-SRF, CTGF prom WT+pLV-EGFP-N-H19+pLV-EGFP$\mathrm{N}-\mathrm{SRF}$ and CTGF prom WT+si-SRF+pLV-EGFP-N-H19. Subsequently, the cells were cultured for an additional $24 \mathrm{~h}$ and then luciferase activity was determined using the Dual-Luciferase Reporter Assay System (Promega).

\section{5-Ethynyl-2'-deoxyuridine (EdU) assay}

Fibroblasts were isolated from co-culture system and inoculated into a 96-well plate at a density of $5 \times 10^{3}$ cells/well. After $6 \mathrm{~h}$, the cells were incubated with EdU culture medium $(100 \mu \mathrm{L} /$ well $)$ for $2 \mathrm{~h}$, then fixed with $4 \%$ paraformaldehyde solution $(100 \mu \mathrm{L} /$ well $)$ at room temperature for $30 \mathrm{~min}$, then rinsed with $2 \mathrm{mg} / \mathrm{mL}$ glycine for $5 \mathrm{~min}$, and finally washed with PBS containing $0.5 \%$ Triton X-100 (100 $\mu \mathrm{L} /$ well $)$ for $10 \mathrm{~min}$. Afterward, the cells were stained with $1 \times$ Apollo and $1 \times$ Hoechst 33342 reaction solution in subdued light at room temperature for $30 \mathrm{~min}$ and added with anti-fluorescence quenching mounting medium (100 $\mu \mathrm{L} /$ well). Cells were then photographed under a fluorescence microscope, and the number of EdU-labeled cells was recorded. Cells with nucleus stained red were regarded as positive. Three visual fields were randomly selected under a microscope to count the number of positive and negative cells.

\section{Terminal deoxynucleotidyl transferase-mediated dUTP-biotin nick end labeling (TUNEL) staining}

Cells were fixed in PBS supplemented with 0.1\% Triton $\mathrm{X}-100$ for $2 \mathrm{~min}$. Then the paraffin sections of wound skin tissues were routinely dewaxed with xylene, rehydrated with gradient ethanol and immersed in $3 \% \mathrm{H}_{2} \mathrm{O}_{2}$ solution at room temperature for $10 \mathrm{~min}$. Next, $50 \mu \mathrm{L}$ of $20 \mu \mathrm{g} / \mathrm{mL}$ protease K (Sigma-Aldrich) was added to the cells for 20-min reaction at room temperature to remove tissue protein. Antigen retrieval was then conducted with citric acid in boiling steamer for $30 \mathrm{~min}$. Thereafter, the cells were added with $50 \mu \mathrm{L}$ TdT enzyme reaction solution and incubated at $37^{\circ} \mathrm{C}$ in subdued light for $1 \mathrm{~h}$, with reaction solution without TdT enzyme as NC. Subsequently, cells and tissue sections were treated with $50 \mu \mathrm{L}$ peroxidaselabeled anti-digoxigenin in darkness at $37^{\circ} \mathrm{C}$ for $30 \mathrm{~min}$ and visualized with DAB (ZSGB-Bio, Beijing, China) for $10 \mathrm{~min}$. Following counterstaining with hematoxylin, the samples were observed and photographed under a microscope (Nikon). Five visual fields were randomly selected to count normal and positive cells.

\section{Hematoxylin-eosin (HE) and Masson's trichrome staining}

The ulcer margin tissues of rats were fixed with $10 \%$ neutral formaldehyde solution for over $24 \mathrm{~h}$. Next, the paraffin sections were dewaxed twice with xylene, stained with hematoxylin for $7 \mathrm{~min}$, dissimilated using hydrochloric acid ethanol, and turned blue with diluted ammonia. 
Then, the sections were stained with eosin for $1 \mathrm{~min}$ and dehydrated with gradient ethanol at the concentrations of 50, 75, 95 and $100 \%$. Then the sections were cleared with xylene and blocked with neutral gum. Subsequently, the sections were observed and photographed under a ten-fold optical microscope to calculate the thickness of epidermis and dermis. Masson's trichrome staining was conducted according to the instructions of the reagent kit (Bogoo Biotechnology, Shanghai, China). ImageJ software was used to determine Masson's trichrome staining area and quantify collagen (Wang et al. 2018).

\section{Immunohistochemistry}

The paraffin sections of rat tissue specimens were dewaxed using xylene, dehydrated with gradient ethanol, immersed in $3 \% \mathrm{H}_{2} \mathrm{O}_{2}$ for $10 \mathrm{~min}$ for endogenous peroxisome elimination, treated with PBS containing $0.1 \%$ Triton $\mathrm{X}-100$ on ice for $10 \mathrm{~min}$ of punching, and reacted with normal goat serum blocking solution (C-0005, Shanghai Haoran Biological Technology, Shanghai, China) at room temperature for $30 \mathrm{~min}$. Next, the sections were probed overnight at $4^{\circ} \mathrm{C}$ with primary antibody CTGF (ab6992, 1:500, Abcam) and then reprobed with secondary antibody goat anti-rabbit IgG (ab6785, 1:1000, Abcam) at $37^{\circ} \mathrm{C}$ for $20 \mathrm{~min}$. Later, the sections were treated with HRP-labeled streptavidin protein working solution (0343-10000U, Imunbio (Beijing) Biotechnology, Beijing, China) at $37^{\circ} \mathrm{C}$ for 20 min, visualized with DAB (ST033, Whiga Biosmart, Guangzhou, Guangdong, China) and counterstained with hematoxylin (PT001, Shanghai Bogoo Biological Technology, Shanghai, China) for 1 min. Subsequently, the sections turned blue in $1 \%$ ammonia water, dehydrated with gradient ethanol at certain concentration, cleared with xylene, mounted with a neutral gum, observed and finally photographed under a microscope. Five random concentration areas were observed in each section, and 100 cells were counted in each area. Cells were graded according to immunoreactive score (IRS) standard (Zhang et al. 2016): IRS $=\mathrm{P} \times \mathrm{I}$; percentage of positive cells: $\mathrm{P}=0$, zero positive cells; $\mathrm{P}=1,1-24 \%$ positive cells; $\mathrm{P}=2,25-49 \%$ positive cells; $\mathrm{P}=3,50-74 \%$ positive cells; and $\mathrm{P}=4,75-100 \%$ positive cells. I stands for staining intensity: $I=0$, non-staining; $I=1$, lightly stained; $I=2$, moderately stained; and $\mathrm{I}=3$, deeply stained.

\section{Statistical analysis}

All data were analyzed using SPSS 21.0 statistical software (IBM.). The measurement data obeying normality distribution and homogeneity of variance were described as mean \pm S.D. Comparisons between two groups were analyzed by unpaired $t$-test, while comparisons among multiple groups were analyzed using one-way ANOVA, followed by Tukey's post hoc tests with corrections for multiple comparisons. ANOVA of repeated measurement with Bonferroni post hoc test was applied for the comparison of data at different time points. A value of $P<0.05$ was statistically significant.

\section{Results}

\section{Culture and identification of fibroblasts in DFU}

After the tissue blocks were cultured for 5-7 days, slight and long shuttle-shaped adherent cells were found in the surrounding area of the tissue blocks. Then, the initial observation was made to compare the morphology of both normal and DFU fibroblasts under an inverted microscope. Morphologically, normal fibroblasts exhibited tight arrangement, radiate, woven or eddy-like shape, overlapping growth, long fusiform or irregular polygon-shaped cell body and short protuberance. There were few intracellular particles without vacuoles. The cell membrane was clear and the cell nucleus was oval (Fig. 1A). However, DFU-derived fibroblasts displayed irregular cell arrangement, larger volume, flat shape, enlarged cytoplasmic area as well as an increase in cytoplasmic protuberances. Intracellular particles and vacuoles were present, while the boundary was not clear and the shape was irregular (Fig. 1B). In addition, immunohistochemistry experiments revealed that positive anti-vimentin (a great number of dark brown particles inside the cytoplasm of fibroblasts) (Fig. 1C) and negative anti-keratin antibody (Fig. 1D) were in conformity with fibroblasts from germ layer. These results demonstrated the successful culture and characterization of fibroblasts in DFU.

\section{CTGF expresses poorly in ulcer tissues of DFU patients and rats}

According to a previous report, CTGF is poorly expressed in diabetic wound tissues (Thomson et al. 2010), leading us to assess CTGF level in ulcer tissues of rats and patients with DFU by conducting RT-qPCR and Western blot analysis. The results (Fig. 2A, B and C) showed that reduced mRNA and protein expression of CTGF were found in ulcer tissues of rats and patients with DFU as compared with controls $(P<0.05)$. Furthermore, immunohistochemistry verified this finding (Fig. 2D and E). Thus, the aforementioned 
A

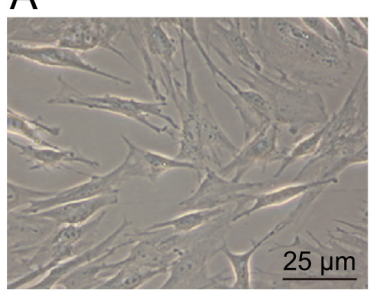

B

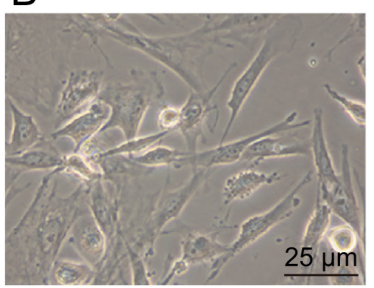

C

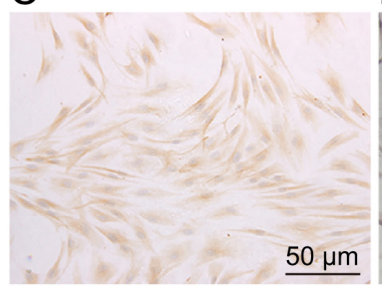

D

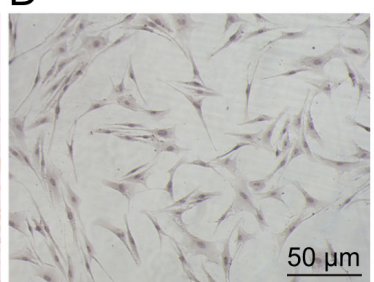

\section{Figure 1}

Culture and identification of fibroblasts collected from ulcer margin of DFU patients or healthy controls. (A) Morphology of normal fibroblasts observed under a brightfield microscope (400×). (B) Morphology of DFU fibroblasts observed under a brightfield microscope (400×). (C) Immunohistochemistry of positive anti-vimentin in fibroblasts (200x). (D) Immunohistochemistry of negative anti-keratin in fibroblasts (200x). In panels A and B, the fibroblasts used in this figure were isolated from ulcer margin of five DFU patients or five healthy controls. Cells from each patient were subjected to two to three repeated experiments. The results in the figure were representative of several experiments.

results substantiated that CTGF was expressed at a low level in ulcer tissues of DFU patients and rats.

\section{CTGF promotes cell proliferation and ECM remodeling but inhibits cell apoptosis by activating the MAPK signaling pathway}

Considering the findings of previous studies and our own results regarding reduced CTGF expression levels in DFU, we performed Western blot analysis, EdU, and TUNEL staining to evaluate the molecular function of CTGF on the expression of proteins related to the MAPK signaling pathway and ECM remodeling, fibroblast proliferation and cell apoptosis. Western blot analysis
(Fig. 3A) revealed that, after overexpression of CTGF, the extent of JNK, ERK and p38 phosphorylation was increased in fibroblasts, suggesting that CTGF upregulation facilitated activation of the MAPK signaling pathway. Moreover, oe-CTGF-transfected fibroblasts showed increased protein expression of collagen II and aggrecan, while the protein expression of MMP3 and MMP13 was decreased $(P<0.05)$, indicating that CTGF upregulation promoted ECM remodeling. In contrast, CTGF silencing resulted in opposite results $(P<0.05)$. The results of EdU assay revealed that cell proliferation was promoted by CTGF overexpression, whereas it was repressed by CTGF inhibition (Fig. 3B and C). Moreover, the results of TUNEL staining demonstrated that CTGF
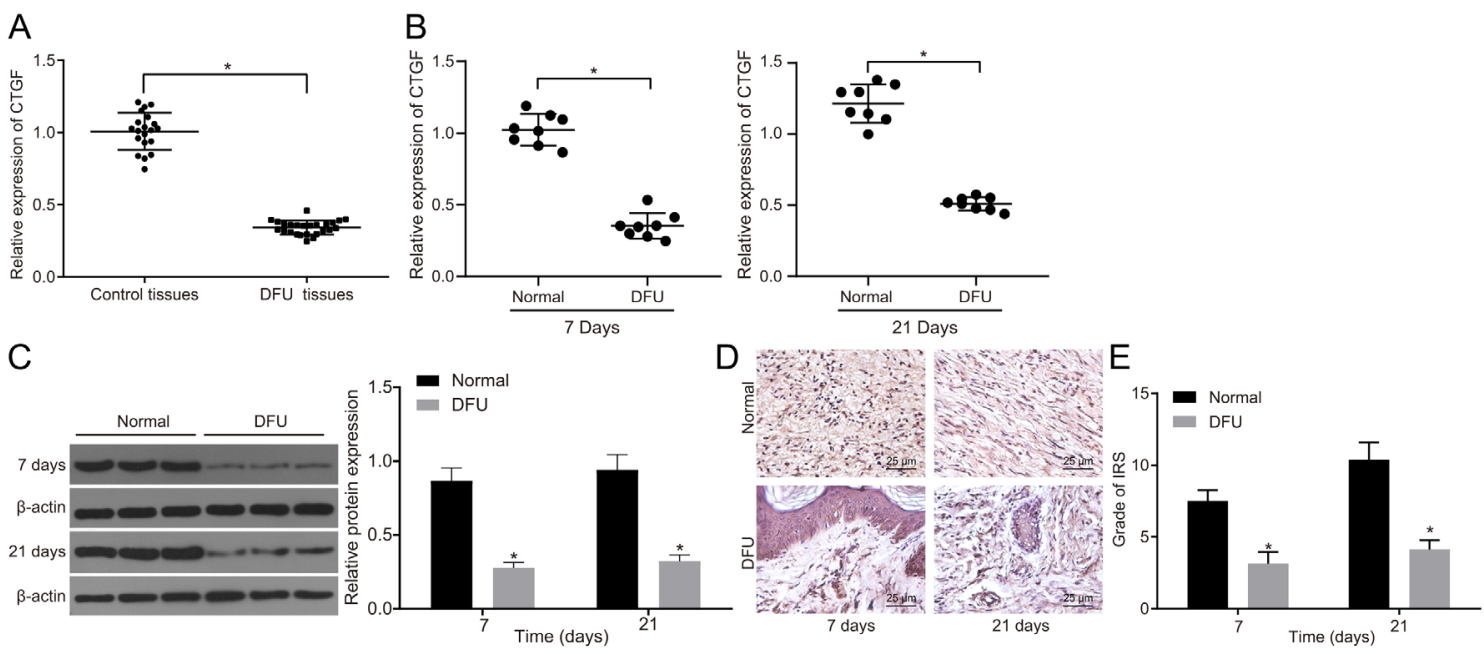

Figure 2

Poor expression of CTGF in ulcer tissues of DFU rats and patients. (A) CTGF level in ulcer tissues of DFU patients detected by RT-qPCR. (B) CTGF level in ulcer tissues of DFU rats detected by RT-qPCR. (C) Western blot analysis of CTGF protein in ulcer tissues of DFU rats. (D and E) Immunohistochemistry of the positive level of CTGF protein in ulcer tissues of DFU rats and the immunoreactive score (IRS) $(400 \times)$. ${ }^{*}<0.05$, compared with control or normal tissues. The results of panel A were enumeration data and expressed as mean \pm s.D. The data were analyzed by independent sample $t$-test. Control tissues: $n=20$; DFU tissues: $n=25$. The results of panels B and D were measurement data and expressed as mean \pm s.D. The data were analyzed by ANOVA of repeated measurement, followed by Tukey's post hoc test. Normal tissues: $n=8$; DFU tissues: $n=8$. Each experiment was repeated at least three times, of which the results of one experiment were selected as a representative. 
overexpression inhibited cell apoptosis while CTGF knockdown induced cell apoptosis (Fig. 3D and E). Taken together, these data suggested that CTGF activated the MAPK signaling pathway, promoting cell proliferation and ECM remodeling while suppressing cell apoptosis.

\section{CTGF enhances wound healing by activating the MAPK signaling pathway in a rat model of DFU}

Subsequently, to evaluate the effect of activated MAPK signaling pathway by CTGF on cell proliferation and apoptosis, ECM remodeling, and wound healing, we established a rat model with DFU to observe wound healing and conducted $\mathrm{HE}$ and Masson's trichrome staining, immunohistochemistry, Western blot analysis and TUNEL staining, respectively, to assess adjacent ulcer tissues, expression level of CTGF, ECM remodeling-related and angiogenesis-related proteins and cell apoptosis. The initial findings showed that overexpressed CTGF promoted wound healing, while MAPK signaling pathway inhibitor reversed this effect (Fig. 4A and B). In addition, $\mathrm{HE}$ and Masson's trichrome staining on rat ulcer tissues transduced with lentivirus for 21 days revealed that overexpressed CTGF resulted in increased thickness of epidermis and dermis and clearly different collagen layer $(P<0.05)$. However, the co-treatment of overexpressed CTGF and MAPK inhibitor reduced the thickness of epidermis and dermis and resulted in a loosely arranged collagen compared with the treatment of overexpressed CTGF alone $(P<0.05)$ (Fig. 4C, D and F). Furthermore, Western blot analysis showed that overexpression of CTGF decreased protein levels of MMP-3 and MMP-13 and increased protein levels of CTGF, collagen II, aggrecan, collagen I, VEGF, TGF- $\beta 1$ and $\alpha$-SMA as well as the extent of JNK, ERK and p38 phosphorylation. However, the co-treatment of oe-CTGF and MAPK inhibitor elevated protein levels of MMP-3 and MMP-13 and reduced protein levels of collagen II, aggrecan, collagen I, VEGF, TGF- $\beta 1$ and $\alpha$-SMA as well as the extent of JNK, ERK and p38 phosphorylation $(P<0.05)$ but did not affect CTGF protein level compared to the treatment of oe-CTGF $(P>0.05$; Fig. 4G). Moreover, immunohistochemistry results revealed that the expression of CTGF was increased in ulcer tissues of rats upon oe-CTGF treatment $(P<0.05)$,
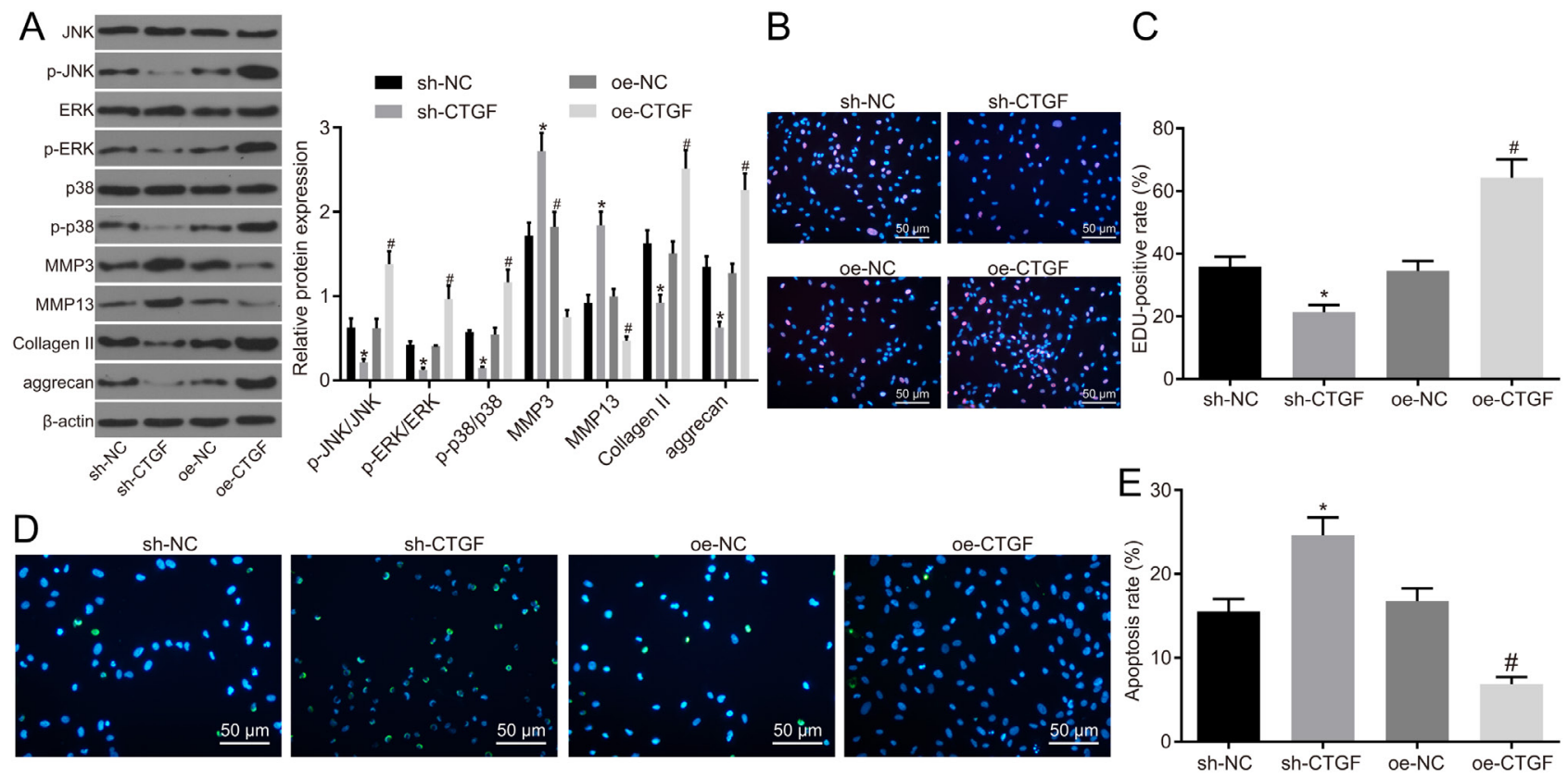

Figure 3

CTGF activates the MAPK signaling pathway, consequently inducing cell proliferation and ECM remodeling while inhibiting cell apoptosis. Cells were treated with elevated or depleted CTGF. (A) Protein bands and levels of collagen II, aggrecan, MMP3, MMP13, JNK, ERK and p38 as well as the extent of JNK, ERK and p38 phosphorylation determined by Western blot analysis. ( $B$ and C) Fibroblast proliferation assessed by EdU assay (200x). (D and E) Fibroblast apoptosis detected by TUNEL staining (200x). $* P<0.05$, compared with cells treated with sh-NC. $\# P<0.05$, compared with cells treated with oe-NC. The aforementioned data were measurement data and expressed as mean \pm s.D. The data were analyzed by ANOVA, followed by Tukey's post hoc test. The experiment was repeated three times. The fibroblasts used in this figure were isolated from ulcer margin of five DFU patients or five healthy controls. 

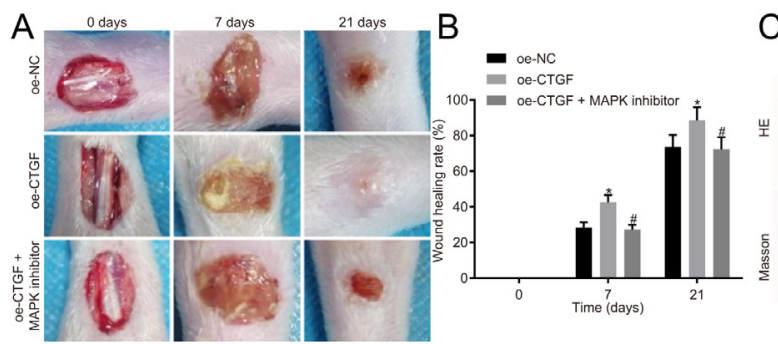

C

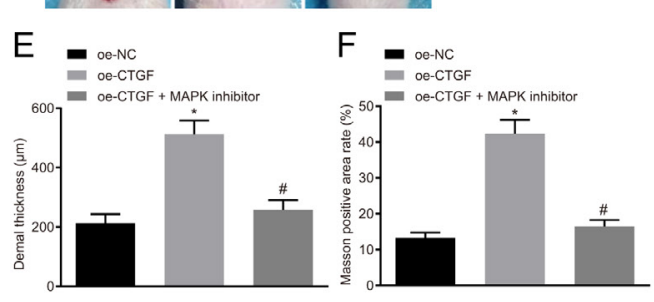

G
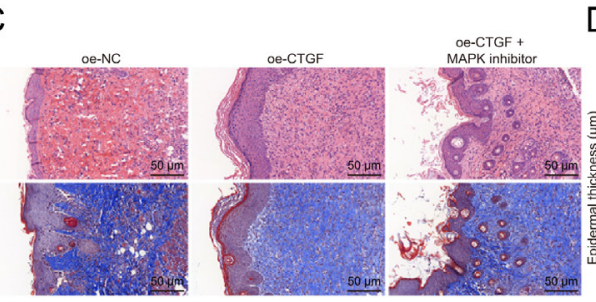

D
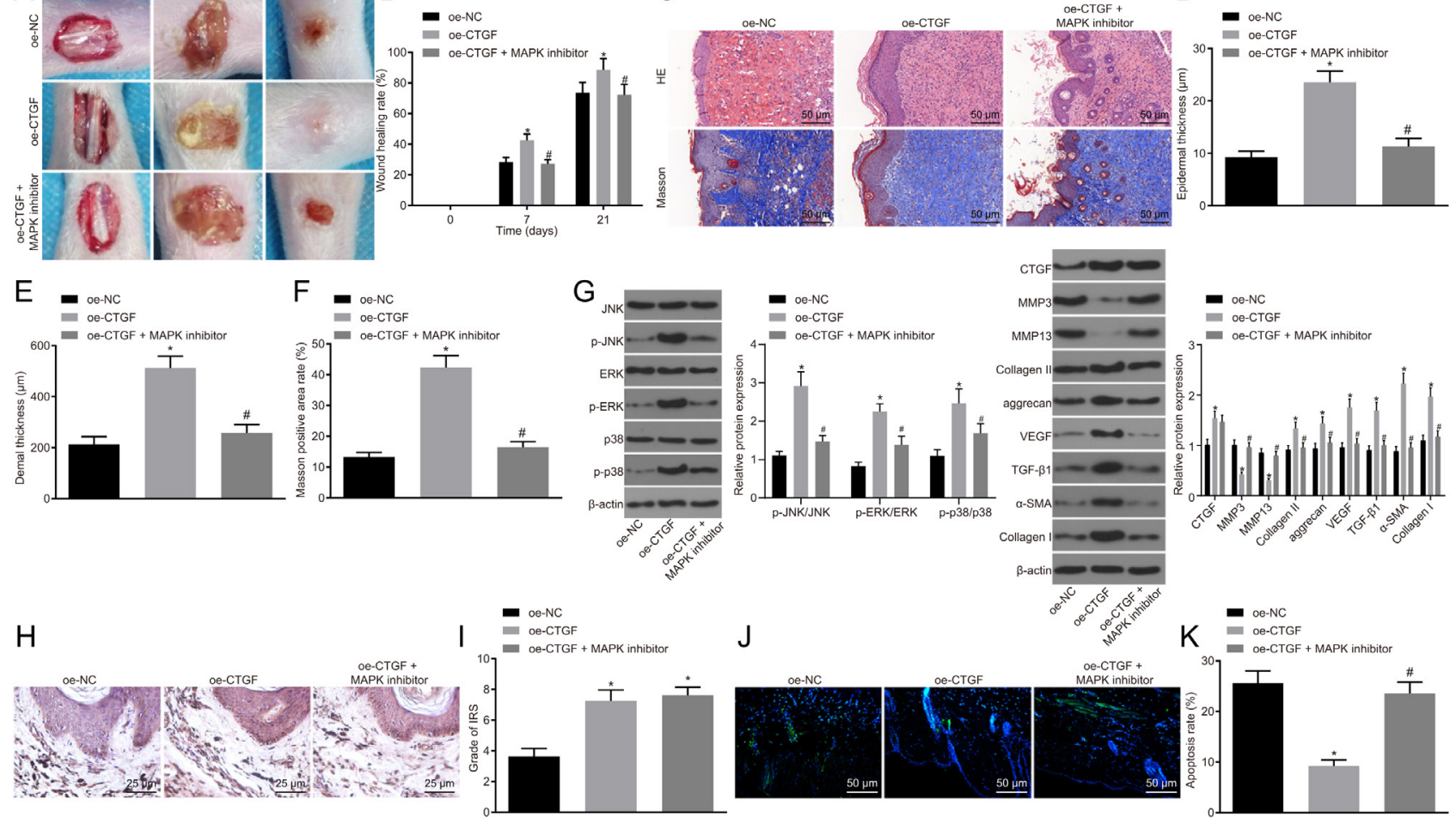

\section{Figure 4}

CTGF overexpression promotes wound healing through activation of the MAPK signaling pathway in a rat model with DFU. DFU rats were injected with cells treated with lentivirus, overexpressed CTGF, or overexpressed CTGF + MAPK inhibitor. (A and B) Wound healing in DFU rats. (C, D, E and F) The thickness of epidermis and dermis and Masson positive area rate measured by HE and Masson's trichrome staining (200x). (G) Western blot analysis of the MAPK signaling pathway-, ECM remodeling- and angiogenesis-related proteins in fibroblasts. (H and I) Immunohistochemistry of CTGF level in ulcer tissues and the immunoreactive score (IRS) $(400 \times)$. ( and K) Cell apoptosis in DFU rats evaluated by TUNEL staining (200x). * $P<0.05$, compared with DFU rats treated with oe-NC. ${ }^{P}<0.05$, compared with DFU rats treated with oe-CTGF. The aforementioned data were measurement data and expressed as mean \pm S.D. The data of panel B were analyzed by ANOVA of repeated measurement, while the data of the remaining panels were analyzed by ANOVA,

followed by Tukey's post hoc test. Normal tissues: $n=8$; DFU tissues: $n=8$.

while it showed no obvious difference in ulcer tissues of rats co-treated with oe-CTGF and MAPK inhibitor when compared to treatment of oe-CTGF alone $(P>0.05$; Fig. $4 \mathrm{H}$ and $\mathrm{I})$. In addition, TUNEL staining demonstrated that cell apoptosis was reduced after the treatment of oe-CTGF, while it was elevated after the co-treatment of oe-CTGF and MAPK inhibitor $(P<0.05)$ (Fig. $4 \mathrm{~J}$ and K). In summary, these data highlighted that overexpression of CTGF activated the MAPK signaling pathway to promote ECM remodeling and angiogenesis, inhibit cell apoptosis and thus enhance wound healing in animal models.

\section{IncRNA H19 recruits SRF to elevate CTGF level}

lncRNAs play an important role in disease progression by altering various gene expressions (Zhang et al. 2019). To locate lncRNAs affecting wound healing in DFU, the LncMAP database was used to retrieve IncRNAs that can affect the transcription of CTGF, which suggested that
IncRNA H19 promoted CTGF transcription through the transcription factor SRF (Fig. 5A). In addition, we retrieved IncRNAs related to diabetes in the LncDisease database (http://www.cuilab.cn/lncrnadisease) and found that lncRNA H19 was involved in diabetic cardiomyopathy, which suggested that IncRNA H19 might also play an important role in DFU. Subsequently, RT-qPCR was employed to detect the expression of IncRNA H19 and SRF in ulcer tissues of DFU rats and patients, the results of which showed that the expression of IncRNA H19 and SRF was decreased in ulcer tissues of DFU rats and patients compared to controls $(P<0.05)$ (Fig. 5B). lncRNA H19 has been flagged as a competing endogenous RNA to mediate CTGF expression by sponging miR-455 in cardiac fibrosis (Huang et al. 2017). We then used a dualluciferase reporter gene assay to assess the effects of SRF and IncRNA H19 on the luciferase activity at the CTGF promoter and found that addition of SRF and lncRNA H19 improved luciferase activity controlled by CTGF promoter 
A
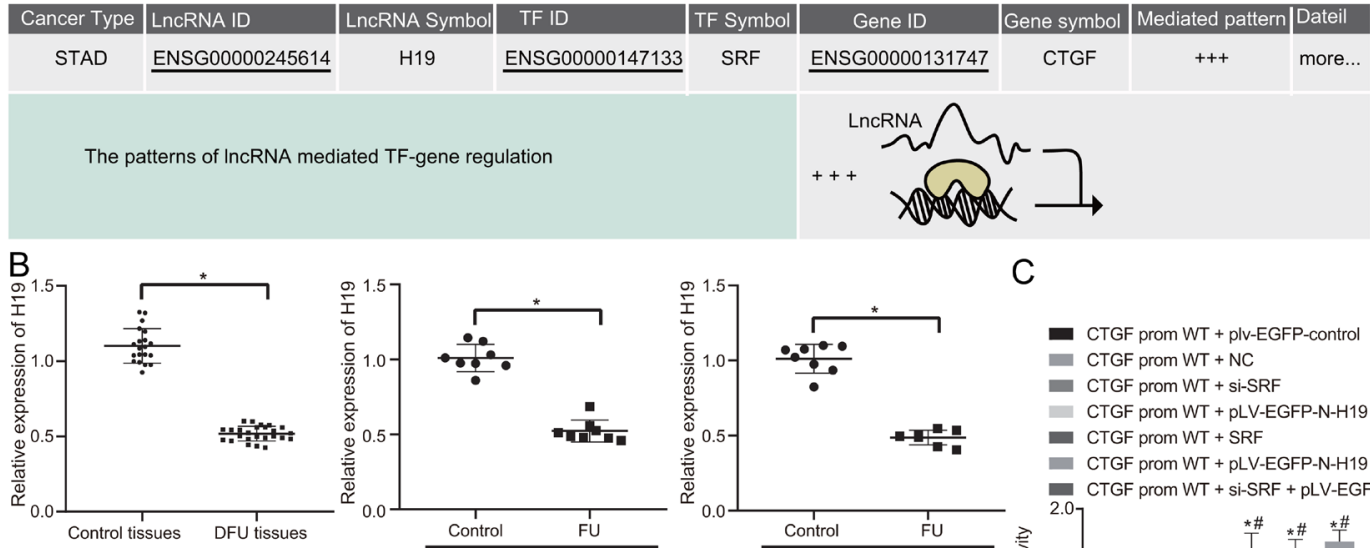

C
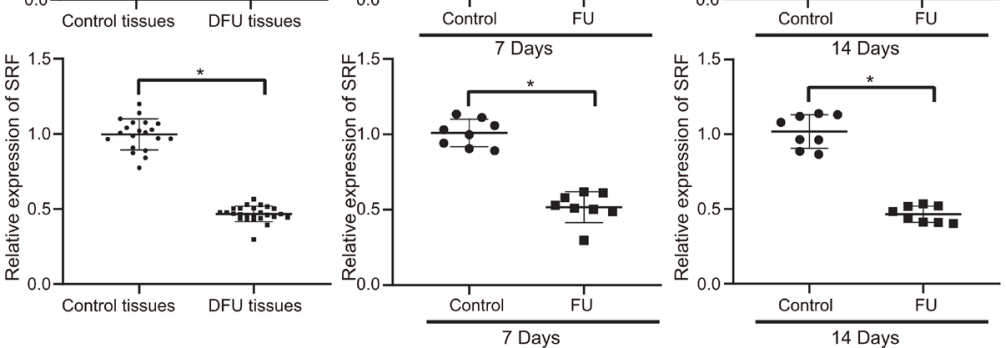
- CTGF prom WT + plv-EGFP-control
CTGF prom WT + si-SRF
CTGF prom WT + pLV-EGFP-N-H19
- CTGF prom WT + pLV-EC
a CTGF prom WT + SRF
- CTGF prom WT + pLV-EGFP-N-H19+ SRF
CTGF prom WT + si-SRF + pLV-EGFP-N-H1
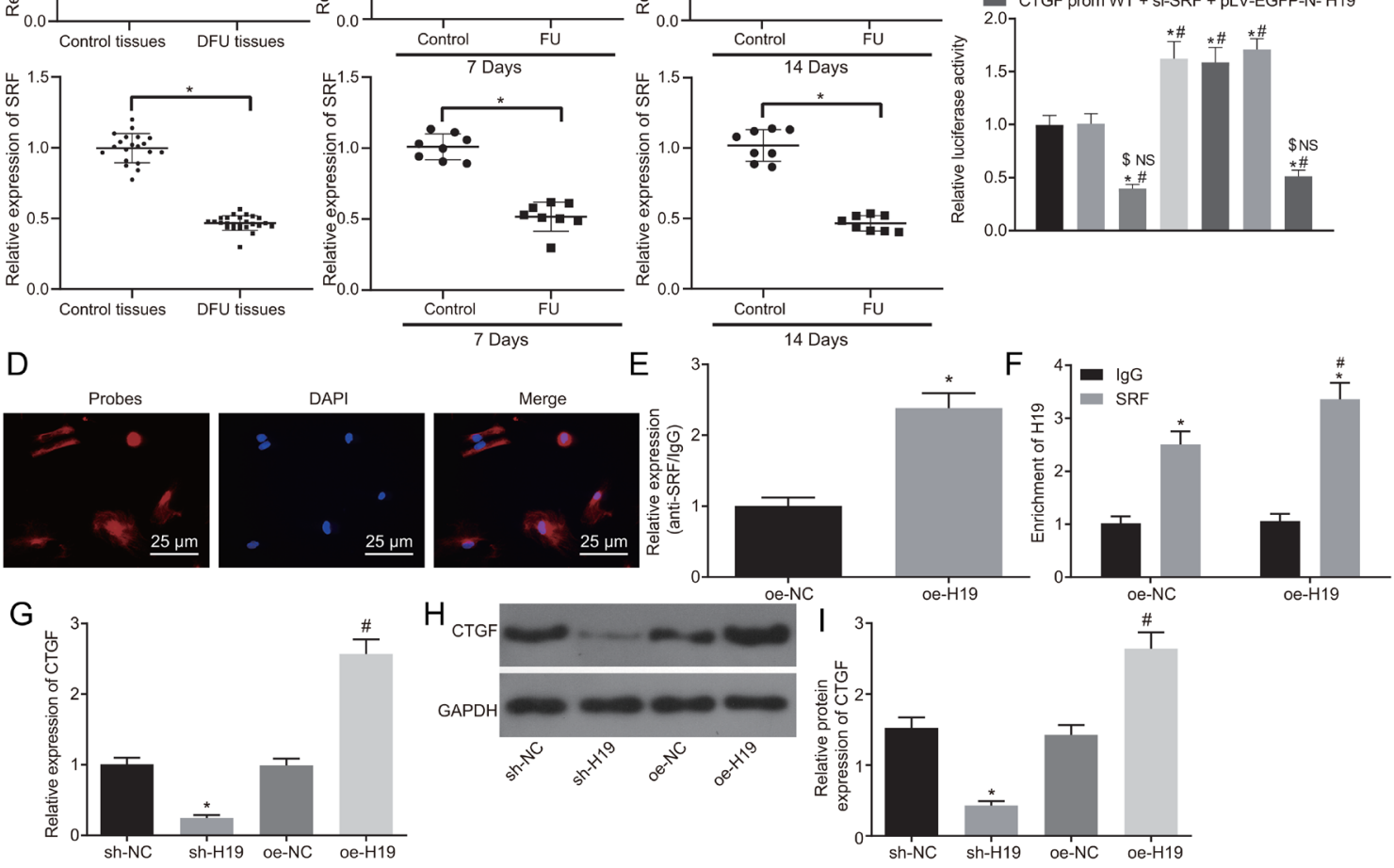

\section{Figure 5}

IncRNA H19 elevates CTGF level by recruiting SRF. (A) IncRNA H19 promoted transcription of CTGF by SRF as demonstrated in LncMAP database and IncRNAs related to diabetes retrieved in the LncDisease database (http://www.cuilab.cn/Incrnadisease). (B) The expression of IncRNA H19 and SRF in ulcer tissues of DFU rats and patients detected by RT-qPCR. ${ }^{*} P<0.05$, compared with control or normal tissues. Patients - control tissues: $n=20$, DFU tissues: $n=25$; rats - normal tissues: $n=8$, DFU tissues: $n=8$. (C) The effects of SRF and IncRNA H19 on luciferase activity of CTGF promoter evaluated by dual-luciferase reporter gene assay. $* P<0.05$, compared with cells treated with CTGF prom WT $+N C$. $\# P<0.05$, compared with cells treated with CTGF prom WT + pLV-EGFP-control. $\$ P<0.05$, compared with cells treated with CTGF prom WT + pLV-EGFP-N-H19, NSP $>0.05$, compared with cells treated with CTGF prom WT + pLV-EGFP-N-H19. (D) The location of IncRNA H19 in fibroblasts detected by FISH (400×). (E) The interaction between SRF and CTGF promoter in fibroblasts assessed by CHIP-PCR. $* P<0.05$, compared with cells treated with oe-NC. (F) The interaction between SRF and IncRNA H19 in fibroblasts measured by RIP. $* P<0.05$, compared with IgG. $\# P<0.05$, compared with cells treated with oe-NC. (G) CTGF level in fibroblasts after IncRNA H19 was elevated or knockdown detected by RT-qPCR. (H and I) Western blot analysis of CTGF protein in fibroblasts after IncRNA H19 was elevated or knockdown. In panel $\mathrm{G}$ and $\mathrm{H}_{\text {, }} * P<0.05$, compared with cells treated with sh-NC. $\# P<0.05$, compared with cells treated with oe-NC. The data were measurement data and expressed as mean \pm S.D. Comparisons between two groups were analyzed by independent sample $t$-test, while comparisons among multiple groups were analyzed using ANOVA, followed by Tukey's post hoc test. The experiment was repeated three times. The fibroblasts used in this figure were isolated from ulcer margin of five DFU patients or five healthy controls.

$(P<0.05)$ (Fig. 5C). Subsequently, a FISH assay detected that IncRNA H19 was mainly localized in the nucleus of fibroblasts (Fig. 5D). Furthermore, to explore whether IncRNA H19 affected CTGF levels directly, ChIP-PCR was carried out to measure the interaction between the CTGF promoter and SRF. The results demonstrated an interaction between the CTGF promoter and SRF $(P<0.05)$ (Fig. 5E). Meanwhile, RIP assay further verified the interaction between SRF and IncRNA H19 $(P<0.05)$ (Fig. 5F). Additionally, the results of RT-qPCR and 
Western blot analysis showed that H19 overexpression elevated the expression of CTGF while IncRNA H19 knockdown reduced CTGF expression $(P<0.05)$ (Fig. 5G, $\mathrm{H}$ and I). These results strongly suggested that CTGF level was elevated after the recruitment of SRF by lncRNA H19.

\section{IncRNA H19 promotes cell proliferation, ECM remodeling and inhibits cell apoptosis by recruiting SRF}

Having demonstrated that lncRNA H19 recruited SRF to elevate CTGF levels, we further performed Western blot analysis, EdU and TUNEL assays to explore the effect of IncRNA H19 on the downstream physiological changes. Western blot analysis illustrated that oe-H19 treatment elevated protein levels of CTGF, collagen II, aggrecan, JNK, ERK and p38 as well as the extent of JNK, ERK and p38 phosphorylation while reducing protein levels of MMP3 and MMP13 $(P<0.05)$. In comparison to co-treatment of oe-H19+sh-NC, co-treatment of oe-H19 and sh-SRF or oe-H19 and sh-CTGF lowered the protein levels of CTGF, collagen II and aggrecan as well as the extent of JNK, ERK and p38 phosphorylation yet elevated protein levels of MMP3 and MMP13 $(P<0.05)$ (Fig. 6A). Additionally, the results of EdU and TUNEL assay showed that overexpression of H19 promoted cell proliferation and inhibited apoptosis, while oe-H19 along with sh-SRF or with sh-CTGF suppressed cell proliferation and enhanced apoptosis in contrast to oe-H19 along with sh-NC $(P<0.05)$ (Fig. 6B and $C)$. Together, these data suggest that lncRNA H19 stimulated cell proliferation and ECM remodeling and attenuated cell apoptosis by recruiting SRF to enhance CTGF expression.

\section{IncRNA H19/SRF/CTGF activates the MAPK signaling pathway to promote wound healing in DFU rats}

Afterward, we evaluated the effect of IncRNA H19/SRF/ CTGF on wound healing in rat models with DFU. Our results showed that overexpression of IncRNA H19 and SRF improved wound healing, which was reversed by perturbing CTGF expression and the addition of MAPK signaling pathway inhibitor $(P<0.05$; Fig. $7 \mathrm{~A}$ and $\mathrm{B})$. Next, after rats' ulcer tissues were injected with lentivirus for 21 days, HE and Masson's trichrome staining were conducted and the results revealed that overexpression of lncRNA H19 and SRF produced increased thickness of epidermis and dermis and clearly different collagen protein layer $(P<0.05)$, while oe-H19 along with sh-CTGF or oe-SRF along with sh-CTGF decreased the thickness of the epidermis and dermis and resulted in a loosely arranged collagen protein layer $(P<0.05)$ (Fig. 7C, D, E, F and G). Afterward, immunohistochemistry demonstrated that the positive level of CTGF protein was elevated after overexpression of IncRNA H19 and SRF, while it was decreased after the treatment of oe-H19 along with sh-CTGF or oe-SRF along with sh-CTGF $(P<0.05$; Fig. $7 \mathrm{H}$ and I). In addition, Western blot analysis determined that overexpression of lncRNA H19 and SRF downregulated protein levels of MMP-3 and MMP-13 and upregulated protein levels of CTGF, collagen II, aggrecan, collagen I, VEGF, TGF- $\beta 1$ and $\alpha$-SMA as well as the extent of JNK, ERK and p38 phosphorylation. An opposite trend was found following treatment of oe-H19 along with sh-CTGF or oe-SRF along with sh-CTGF or oe-H19 along with MAPK inhibitor $(P<0.05$; Fig. $7 \mathrm{~J})$, which suggested that the effect of CTGF was reversed by MAPK inhibitor. At last, the results of TUNEL staining illustrated that overexpression of IncRNA H19 and SRF induced a decline in cell apoptosis, while oe-H19 along with sh-CTGF or oe-SRF along with sh-CTGF led to an increase in cell apoptosis $(P<0.05$; Fig. 7K and L). To sum up, IncRNA H19 recruited SRF to elevate CTGF level and activated the MAPK signaling pathway, thus inhibiting cell apoptosis and promoting ECM remodeling, angiogenesis, and subsequent wound healing in rat models with DFU.

\section{Discussion}

DFU is a heavy health burden for both individuals and the society, which is accompanied by severe pain, high risk of recurrence, amputation and increased mortality (Monteiro-Soares et al. 2012). Currently, many regulatory mechanisms involved in the progression of DFU. For instance, CTGF, a critical fibrogenic factor, is associated with the thickening of capillary basement membrane and proliferation of fibrovascular tissues in proliferative diabetic retinopathy (Zhang et al. 2010). IncRNAs have been reported to be dynamically modulated, tissue-specific and aberrantly expressed in type 2 diabetes (Moran et al. 2012). The aim of the present study was to evaluate the role of IncRNA H19 and CTGF in DFU. Collectively, lncRNA H19 promoted wound healing in DFU via elevating CTGF by recruiting SRF and subsequently activating the MAPK signaling pathway.

One important finding of our study was that CTGF was poorly expressed in the ulcer tissues of DFU rats and patients. CTGF is a critical member of the CCN matricellular protein family, which interacts with various membrane proteins and regulates cell adhesion in 

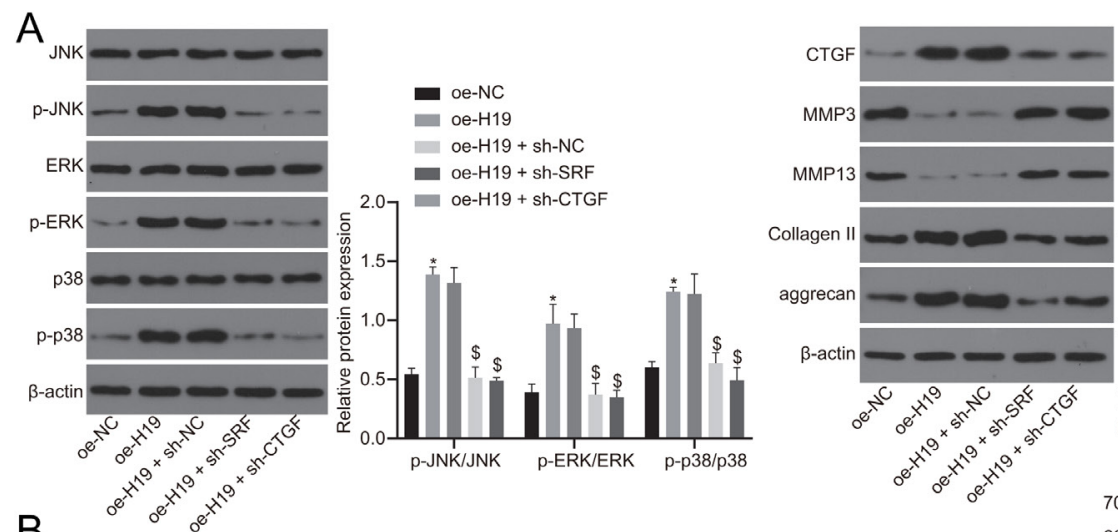

$$
\begin{aligned}
& \text { oe-NC } \\
\text { oe-H19 } & \text { oe-H19 + sh-NC } \\
& \text { oe-H19 + sh-SRF } \\
& \text { oe-H19 + sh-CTGF }
\end{aligned}
$$
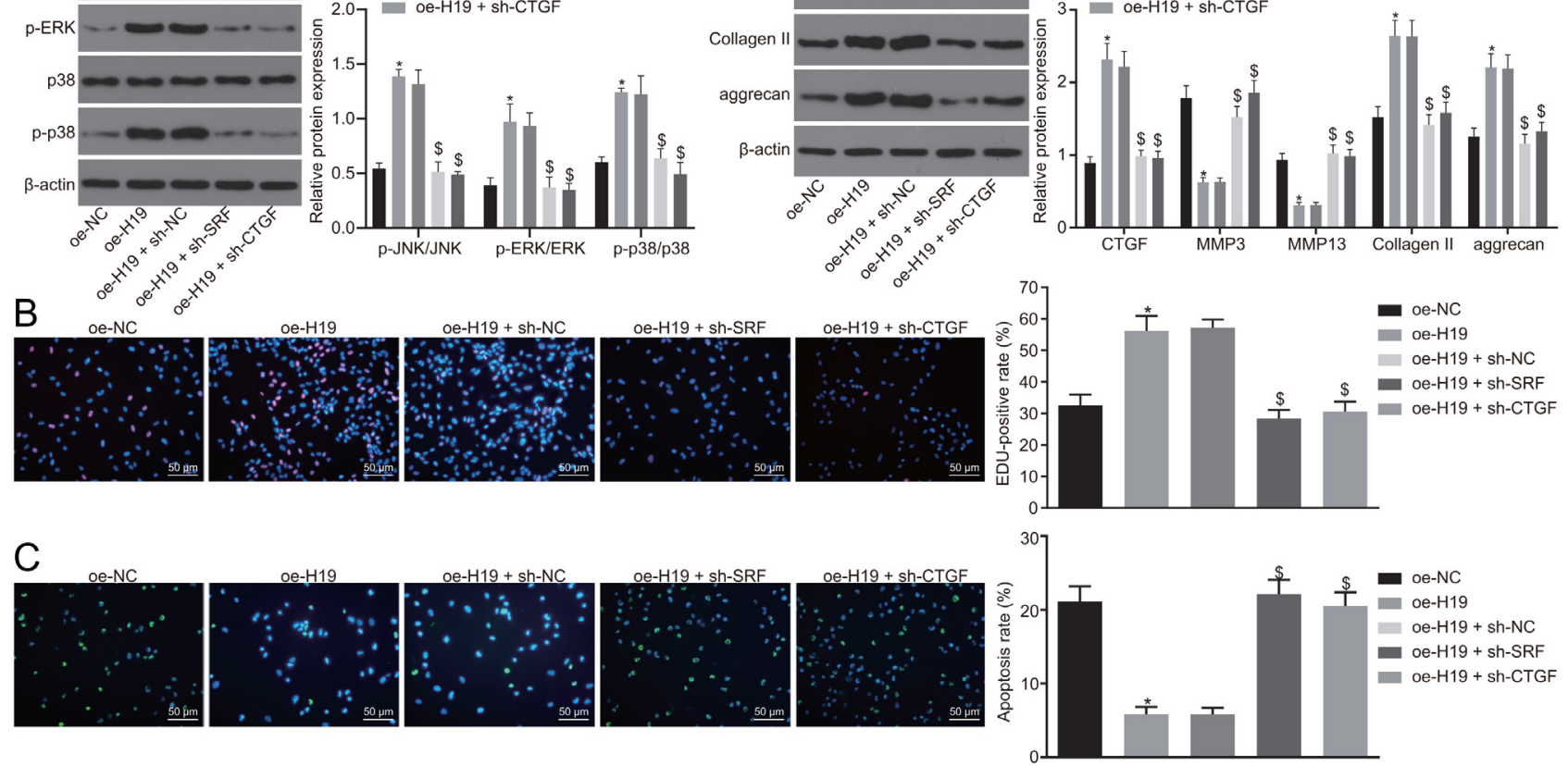

\section{Figure 6}

IncRNA H19 enhances cell proliferation and ECM remodeling while suppressing cell apoptosis through recruiting SRF to promote CTGF expression. Fibroblasts were treated with oe-H19, sh-SRF or co-treated with oe-H19 and sh-SRF or oe-H19 and sh-CTGF. (A) Western blot analysis of CTGF, collagen II, aggrecan, MMP3, MMP13, JNK, ERK and p38 proteins as well as extent of JNK, ERK and p38 phosphorylation in cells. (B) Cell proliferation assessed by EdU assay (200×). (C) Cell apoptosis measured by TUNEL assay (200×). ${ }^{*} P<0.05$, compared with cells treated with oe-NC. \$Compared with cells co-treated with oe-H19 and sh-NC. The data were measurement data and expressed as mean \pm s.D. Comparisons among multiple groups were analyzed using ANOVA, followed by Tukey's post hoc test. The experiment was repeated three times. The fibroblasts used in this figure were isolated from ulcer margin of five DFU patients or five healthy controls.

multiple cell types including fibroblasts, myofibroblasts, endothelial and epithelial cells (Ball et al. 2003, Mason 2013). Mutation or deletions in the CTGF gene have been shown to lead to the development and progression of diabetic nephropathy (Wang et al. 2015). In addition, a significant decrease in glomerular mRNA levels of CTGF in patients with diabetic nephropathy was found in a previous study (Baelde et al. 2007). The presence of CTGF and periostin scaffolds induces the acceleration of the wound healing response in the skin of diabetic mice (Elliott et al. 2019). However, the level of CTGF in diabetic wounds turns to be slightly different. In the immunohistochemistry experiments conducted by Thomson SE, a lower CTGF level has been detected in diabetic wound tissue at 4 weeks when compared with controls (Thomson et al. 2010). Besides, CTGF has been identified as a target for drug therapy in diabetes and often displays a high level in diabetic patients (Leask 2010). Therefore, more investigations are needed in order to clarify the molecular function of CTGF in various diabetes complications. Taken together, the aforementioned findings provided evidence that CTGF is poorly expressed in DFU.

Additionally, overexpression of CTGF activated the MAPK signaling pathway to promote fibroblast proliferation, ECM remodeling and angiogenesis while inhibiting cell apoptosis, as evidenced by the elevated extent of JNK, ERK and p38 phosphorylation as well as expression of collagen II, aggrecan, collagen I, VEGF, TGF- $\beta 1$ and $\alpha$-SMA and decreased levels of MMP3 and MMP13. ERK, JNK and p38 are important components of the MAPKs pathway, where they modulate various cellular functions such as autophagy, ciliogenesis and protein secretion (Colecchia et al. 2012, Lanna et al. 2017). Collagen proteins including collagen I and II are known as prominent elements of extracellular matrices (Barnes et al. 2007, Goldberga et al. 2018). Aggrecan, a dominating proteoglycan in cartilage, imparts the ability to resist compression and load-bearing in the tissues (Stanton et al. 2005). ECM is a dominant element of any multicellular 

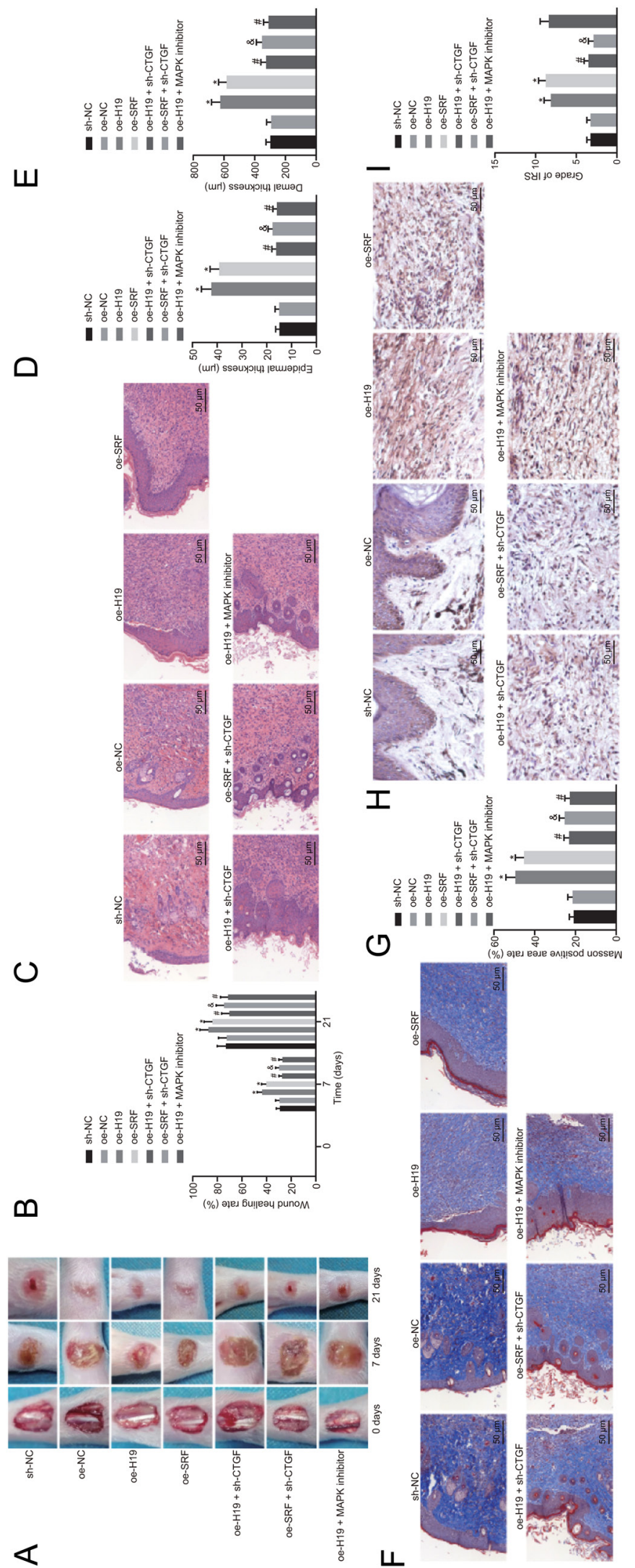
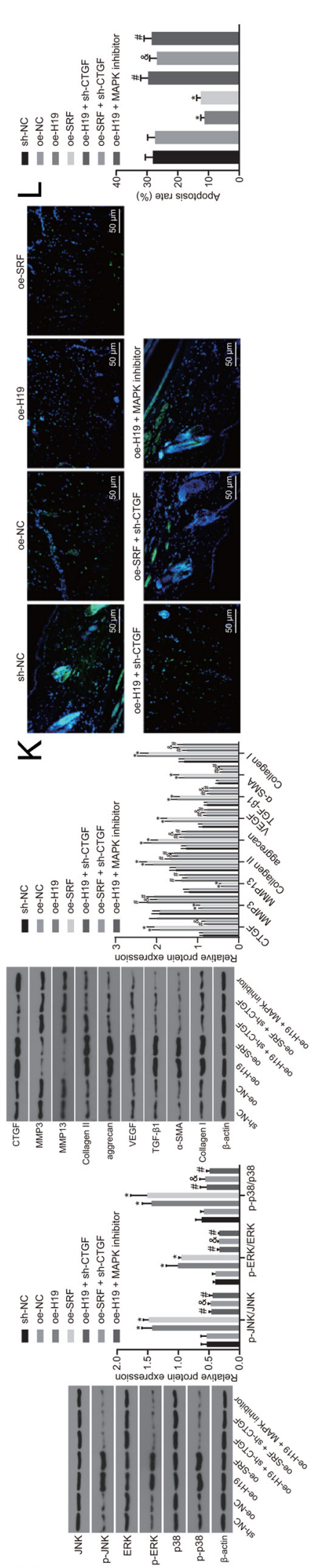

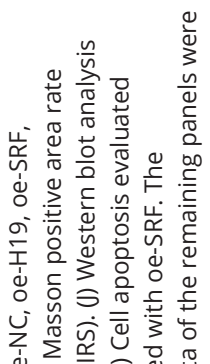

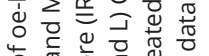

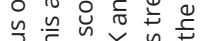

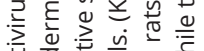

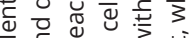

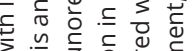

उद्ध है.

要

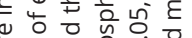

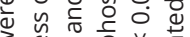

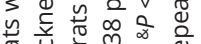

政

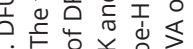

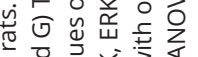

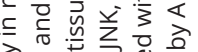

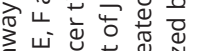

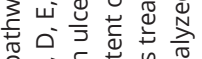

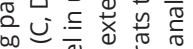

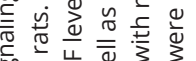

品

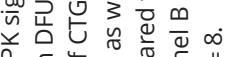

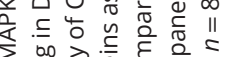

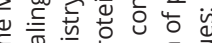

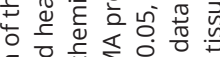

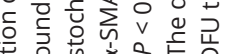

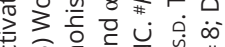

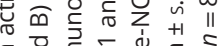

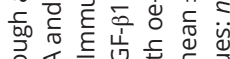

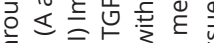

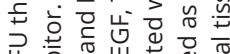

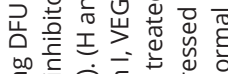

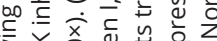

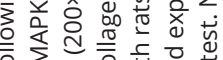

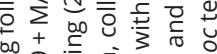

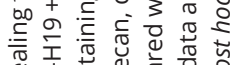

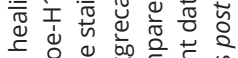

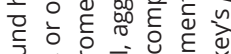

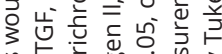

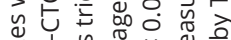

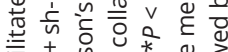

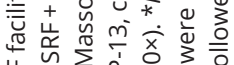

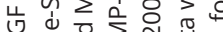

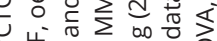

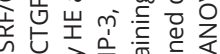

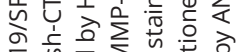

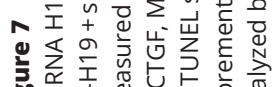


organism and confers tissue organization and structural support by building a complicated proteinaceous network, containing epi- and endothelial cells, fibroblasts, and collagen proteins (Frangogiannis 2012). CTGF has been shown to induce corneal angiogenesis and to stimulate excess ECM production in pathological fibrosis (HallGlenn et al. 2012). In addition, CTGF can cause migration, proliferation and ECM overproduction in Keloid fibroblasts (Luo et al. 2017). Moreover, CTGF is correlated with the promotion of hypertrophic scar fibroblast proliferation (Liu et al. 2018). CTGF/Hcs24 promotes proliferation and differentiation of chondrocytes through activation of the p38/MAPK and ERK/MAPK signaling pathways (Yosimichi et al. 2001). Vaccarin is able to induce angiogenesis and accelerate wound healing in vivo via activation of the MAPK/ERK signaling pathway (Hou et al. 2020). Collectively, CTGF-dependent MAPK signaling pathway activation was involved in the wound healing upon DFU.

Moreover, we found that IncRNA H19 recruited SRF to upregulate CTGF levels, thus promoting wound healing in DFU. SRF is a transcription factor induced by neuronal activity and links to immediate and long-term coping mechanism associated with acute stress (Zimprich et al. 2017). SRF has been revealed to display a lower level in the cavernous tissue of diabetic rats (He et al. 2012). Besides, SRF plays a significant role in the differentiation of myofibroblast during experimental oesophageal ulcer healing in rats (Chai et al. 2007). According to a recent report, both CTGF and SRF exert critical effects on idiopathic pulmonary fibrosis (Plantier et al. 2016). CTGF has been associated with tissue repair and wound healing (Sonnylal et al. 2010). In addition, CTGF treated diabetic wounds show an accelerated healing rate as compared with

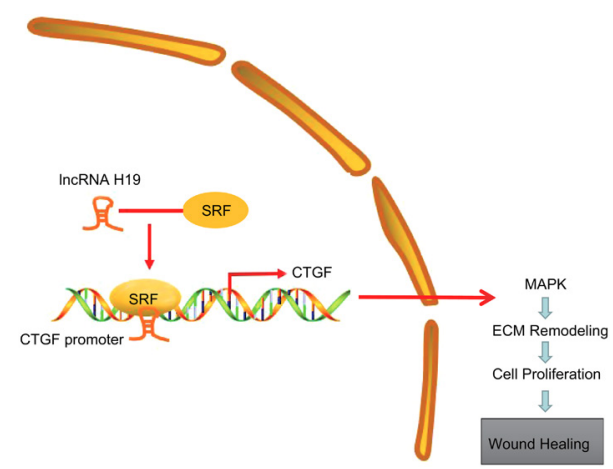

\section{Figure 8}

The mechanism graph of the regulatory network of the IncRNA H19/SRF/ CTGF axis in wound healing following DFU. IncRNA H19 recruited SRF to increase CTGF expression and consequently induced activated MAPK signaling pathway, thus repressing cell apoptosis and enhancing ECM remodeling, angiogenesis, ultimately promoting wound healing in DFU. vehicle-treated diabetic wounds (Henshaw et al. 2015). Meanwhile, another study has shown that CTGF-coated acellular dermal matrix scaffolds facilitate diabetic wound healing through elevation of fibronectin by PKC signaling pathway (Yan et al. 2018). IncRNA H19 has been reported to antagonize miR-455 and consequently increase CTGF expression in cardiac fibroblasts (Huang et al. 2017). IncRNA H19 has been shown to enhance fibroblast activation by activating the HIF-1 $\alpha$ signaling pathway in fibroblasts and promote wound healing in diabetic mice (Guo et al. 2018). Furthermore, mesenchymal stem-cell-derived exosomes overexpressing lncRNA H19 promote fibroblast proliferation and migration, as well as suppress apoptosis and inflammation, whichstimulates the wound healing process in DFU ( $\mathrm{Li}$ et al. 2020). These data collectively increasingly substantiated a functional role for lncRNA H19 in regulating CTGF in human diabetic foot ulcers.

Taken together, the lncRNA H19/SRF/CTGF axis is a novel regulatory network in the wound healing following DFU (Fig. 8). Our findings offer new perspectives for accelerating wound healing of DFU and suggest a new target for the translational value of IncRNA H19/SRF/ CTGF in DFU wound healing. However, the intrinsic functional mechanisms of IncRNA H19/SRF/CTGF in DFU still remain to be further examined and verified. Besides, despite interesting promises for the prognostic and therapeutic approaches in DFU, clinical experiments of fully developed lncRNA H19/SRF/CTGF-based therapeutic treatment should be carried out in the future.

Declaration of interest

The authors declare that there is no conflict of interest that could be perceived as prejudicing the impartiality of the research reported.

\section{Funding}

This study was supported by the National Natural Science Foundation of China (Nos 81501737, 81871562, 81800739).

\section{Author contribution statement}

Bo Li, Yue Zhou, Jing Chen, Tingting Wang, Zhijuan Li, Yili Fu, Changlong Bi and Aixia Zhai designed the study. Bo Li, Yue Zhou, Jing Chen and Tingting Wang collated the data, carried out data analyses and produced the initial draft of the manuscript. Zhijuan Li, Yili Fu, Changlong Bi and Aixia Zhai contributed to drafting the manuscript. All authors have read and approved the final submitted manuscript.

\section{Acknowledgements}

The authors acknowledge and appreciate their colleagues for their valuable efforts and comments on this paper. 


\section{References}

Armstrong DG, Boulton AJM \& Bus SA 2017 Diabetic foot ulcers and their recurrence. New England Journal of Medicine 376 2367-2375. (https://doi.org/10.1056/NEJMra1615439)

Arthur JS \& Ley SC 2013 Mitogen-activated protein kinases in innate immunity. Nature Reviews: Immunology 13 679-692. (https://doi. org/10.1038/nri3495)

Baelde HJ, Eikmans M, Lappin DW, Doran PP, Hohenadel D, Brinkkoetter PT, van der Woude FJ, Waldherr R, Rabelink TJ, de Heer E, et al. 2007 Reduction of VEGF-A and CTGF expression in diabetic nephropathy is associated with podocyte loss. Kidney International 71 637-645. (https://doi.org/10.1038/sj.ki.5002101)

Ball DK, Rachfal AW, Kemper SA \& Brigstock DR 2003 The heparinbinding $10 \mathrm{kDa}$ fragment of connective tissue growth factor (CTGF) containing module 4 alone stimulates cell adhesion. Journal of Endocrinology 176 R1-R7. (https://doi.org/10.1677/joe.0.176r001)

Barnes CP, Sell SA, Boland ED, Simpson DG \& Bowlin GL 2007 Nanofiber technology: designing the next generation of tissue engineering scaffolds. Advanced Drug Delivery Reviews 59 1413-1433. (https://doi.org/10.1016/j.addr.2007.04.022)

Blumberg SN, Berger A, Hwang L, Pastar I, Warren SM \& Chen W 2012 The role of stem cells in the treatment of diabetic foot ulcers. Diabetes Research and Clinical Practice 96 1-9. (https://doi. org/10.1016/j.diabres.2011.10.032)

Chai J, Norng M, Tarnawski AS \& Chow J 2007 A critical role of serum response factor in myofibroblast differentiation during experimental oesophageal ulcer healing in rats. Gut 56 621-630. (https://doi. org/10.1136/gut.2006.106674)

Colecchia D, Strambi A, Sanzone S, Iavarone C, Rossi M, Dall'Armi C, Piccioni F, Verrotti di Pianella A \& Chiariello M 2012 MAPK15/ERK8 stimulates autophagy by interacting with LC3 and GABARAP proteins. Autophagy 8 1724-1740. (https://doi.org/10.4161/auto.21857)

Dumville JC, Soares MO, O'Meara S \& Cullum N 2012 Systematic review and mixed treatment comparison: dressings to heal diabetic foot ulcers. Diabetologia 55 1902-1910. (https://doi.org/10.1007/s00125012-2558-5)

Elliott CG, Wang J, Walker JT, Michelsons S, Dunmore-Buyze J, Drangova M, Leask A \& Hamilton DW 2019 Periostin and CCN2 scaffolds promote the wound healing response in the skin of diabetic mice. Tissue Engineering. Part A 25 1326-1339. (https://doi. org/10.1089/ten.TEA.2018.0268)

Fan W, Peng Y, Liang Z, Yang Y \& Zhang J 2019 A negative feedback loop of H19/miR-675/EGR1 is involved in diabetic nephropathy by downregulating the expression of the vitamin D receptor. Journal of Cellular Physiology 234 17505-17513. (https://doi.org/10.1002/ jcp.28373)

Frangogiannis NG 2012 Matricellular proteins in cardiac adaptation and disease. Physiological Reviews 92 635-688. (https://doi.org/10.1152/ physrev.00008.2011)

Gan J, Liu C, Li H, Wang S, Wang Z, Kang Z, Huang Z, Zhang J, Wang C, Lv D, et al. 2019 Accelerated wound healing in diabetes by reprogramming the macrophages with particle-induced clustering of the mannose receptors. Biomaterials 219 119340. (https://doi. org/10.1016/j.biomaterials.2019.119340)

Goldberga I, Li R \& Duer MJ 2018 Collagen structure-function relationships from solid-state NMR spectroscopy. Accounts of Chemical Research 51 1621-1629. (https://doi.org/10.1021/acs. accounts.8b00092)

Goldin A, Beckman JA, Schmidt AM \& Creager MA 2006 Advanced glycation end products: sparking the development of diabetic vascular injury. Circulation 114 597-605. (https://doi.org/10.1161/ CIRCULATIONAHA.106.621854)

Goodson EJ 2016 Earl Wiley Renfroe: man of fortitude and orthodontist extraordinaire. American Journal of Orthodontics and Dentofacial
Orthopedics 150 217-219. (https://doi.org/10.1016/j. ajodo.2016.05.001)

Goyal N, Tiwary S, Kesharwani D \& Datta M 2019 Long non-coding RNA H19 inhibition promotes hyperglycemia in mice by upregulating hepatic FoxO1 levels and promoting gluconeogenesis. Journal of Molecular Medicine 97 115-126. (https://doi.org/10.1007/ s00109-018-1718-6)

Guo JR, Yin L, Chen YQ, Jin XJ, Zhou X, Zhu NN, Liu XQ, Wei HW \& Duan LS 2018 Autologous blood transfusion augments impaired wound healing in diabetic mice by enhancing lncRNA H19 expression via the HIF-1 $\alpha$ signaling pathway. Cell Communication and Signaling 16 84. (https://doi.org/10.1186/s12964-018-0290-6)

Hall-Glenn F, De Young RA, Huang BL, van Handel B, Hofmann JJ, Chen TT, Choi A, Ong JR, Benya PD, Mikkola H, et al. 2012 CCN2/ connective tissue growth factor is essential for pericyte adhesion and endothelial basement membrane formation during angiogenesis. PLoS ONE 7 e30562. (https://doi.org/10.1371/journal. pone.0030562)

He SH, Wei AY, Yang Y, Hu YW, Luo XG, Liu Y \& Zhang T 2012 Reduced expression of myocardin and serum response factor in the cavernous tissue of diabetic rats. Andrologia 44 (Supplement 1) 518-522. (https://doi.org/10.1111/j.1439-0272.2011.01218.x)

Henshaw FR, Boughton P, Lo L, McLennan SV \& Twigg SM 2015 Topically applied connective tissue growth factor/CCN2 improves diabetic preclinical cutaneous wound healing: potential role for CTGF in human diabetic foot ulcer healing. Journal of Diabetes Research 2015 236238. (https://doi.org/10.1155/2015/236238)

Hon CC, Ramilowski JA, Harshbarger J, Bertin N, Rackham OJ, Gough J, Denisenko E, Schmeier S, Poulsen TM, Severin J, et al. 2017 An atlas of human long non-coding RNAs with accurate 5 ' ends. Nature $\mathbf{5 4 3}$ 199-204. (https://doi.org/10.1038/nature21374)

Hou B, Cai W, Chen T, Zhang Z, Gong H, Yang W \& Qiu L 2020 Vaccarin hastens wound healing by promoting angiogenesis via activation of MAPK/ERK and PI3K/AKT signaling pathways in vivo. Acta Cirurgica Brasileira 34 e201901202. (https://doi.org/10.1590/ s0102-865020190120000002)

Huang ZW, Tian LH, Yang B \& Guo RM 2017 Long noncoding RNA H19 acts as a competing endogenous RNA to mediate CTGF expression by sponging miR-455 in cardiac fibrosis. DNA and Cell Biology 36 759-766. (https://doi.org/10.1089/dna.2017.3799)

Hunt KJ, Jaffa MA, Garrett SM, Luttrell DK, Lipson KE, Lopes-Virella MF, Luttrell LM, Jaffa AA \& Investigators V 2018 Plasma connective tissue growth factor (CTGF/CCN2) levels predict myocardial infarction in the veterans affairs diabetes trial (VADT) cohort. Diabetes Care 41 840-846. (https://doi.org/10.2337/dc17-2083)

Jin W, Goldfine AB, Boes T, Henry RR, Ciaraldi TP, Kim EY, Emecan M, Fitzpatrick C, Sen A, Shah A, et al. 2011 Increased SRF transcriptional activity in human and mouse skeletal muscle is a signature of insulin resistance. Journal of Clinical Investigation 121 918-929. (https://doi.org/10.1172/JCI41940)

Kranke P, Bennett MH, Martyn-St James M, Schnabel A, Debus SE \& Weibel S 2015 Hyperbaric oxygen therapy for chronic wounds. Cochrane Database of Systematic Reviews 6 CD004123. (https://doi. org/10.1002/14651858.CD004123.pub4)

Lanna A, Gomes DC, Muller-Durovic B, McDonnell T, Escors D, Gilroy DW, Lee JH, Karin M \& Akbar AN 2017 A sestrin-dependent Erk-Jnk-p38 MAPK activation complex inhibits immunity during aging. Nature Immunology 18 354-363. (https://doi.org/10.1038/ ni.3665)

Lawrence MC, Jivan A, Shao C, Duan L, Goad D, Zaganjor E, Osborne J, McGlynn K, Stippec S, Earnest S, et al. 2008 The roles of MAPKs in disease. Cell Research 18 436-442. (https://doi.org/10.1038/ cr.2008.37)

Leask A 2010 Getting to the heart of the matter: CCN2 plays a role in cardiomyocyte hypertrophy. Journal of Cell Communication and Signaling 4 73-74. (https://doi.org/10.1007/s12079-009-0061-7) 
Li B, Luan S, Chen J, Zhou Y, Wang T, Li Z, Fu Y, Zhai A \& Bi C 2020 The MSC-derived exosomal lncRNA H19 promotes wound healing in diabetic foot ulcers by upregulating PTEN via microRNA-152-3p. Molecular Therapy: Nucleic Acids 19 814-826. (https://doi. org/10.1016/j.omtn.2019.11.034)

Liu F, Chen WW, Li Y, Zhang JQ \& Zheng QB 2018 MiR-6836-3p promotes proliferation of hypertrophic scar fibroblasts by targeting CTGF. European Review for Medical and Pharmacological Sciences 22 4069-4074. (https://doi.org/10.26355/eurrev_201807_15396)

Loots MA, Lamme EN, Zeegelaar J, Mekkes JR, Bos JD \& Middelkoop E 1998 Differences in cellular infiltrate and extracellular matrix of chronic diabetic and venous ulcers versus acute wounds. Journal of Investigative Dermatology 111 850-857. (https://doi. org/10.1046/j.1523-1747.1998.00381.x)

Luo L, Li J, Liu H, Jian X, Zou Q, Zhao Q, Le Q, Chen H, Gao X \& He C 2017 Adiponectin is involved in connective tissue growth factorinduced proliferation, migration and overproduction of the extracellular matrix in keloid fibroblasts. International Journal of Molecular Sciences 18 1044. (https://doi.org/10.3390/ijms18051044)

Mason RM 2013 Fell-Muir lecture: connective tissue growth factor (CCN2) - a pernicious and pleiotropic player in the development of kidney fibrosis. International Journal of Experimental Pathology $\mathbf{9 4}$ 1-16. (https://doi.org/10.1111/j.1365-2613.2012.00845.x)

Monteiro-Soares M, Boyko EJ, Ribeiro J, Ribeiro I \& Dinis-Ribeiro M 2012 Predictive factors for diabetic foot ulceration: a systematic review. Diabetes/Metabolism Research and Reviews 28 574-600. (https://doi.org/10.1002/dmrr.2319)

Moran I, Akerman I, van de Bunt M, Xie R, Benazra M, Nammo T, Arnes L, Nakic N, Garcia-Hurtado J, Rodriguez-Segui S, et al. 2012 Human beta cell transcriptome analysis uncovers IncRNAs that are tissue-specific, dynamically regulated, and abnormally expressed in type 2 diabetes. Cell Metabolism 16 435-448. (https://doi. org/10.1016/j.cmet.2012.08.010)

Pi L, Robinson PM, Jorgensen M, Oh SH, Brown AR, Weinreb PH, Trinh TL, Yianni P, Liu C, Leask A, et al. 2015 Connective tissue growth factor and integrin alphavbeta6: a new pair of regulators critical for ductular reaction and biliary fibrosis in mice. Hepatology 61 678-691. (https://doi.org/10.1002/hep.27425)

Plantier L, Renaud H, Respaud R, Marchand-Adam S \& Crestani B 2016 Transcriptome of cultured lung fibroblasts in idiopathic pulmonary fibrosis: meta-analysis of publically available microarray datasets reveals repression of inflammation and immunity pathways. International Journal of Molecular Sciences 17 2091. (https://doi. org/10.3390/ijms17122091)

Shahbazian H, Yazdanpanah L \& Latifi SM 2013 Risk assessment of patients with diabetes for foot ulcers according to risk classification consensus of International Working Group on Diabetic Foot (IWGDF). Pakistan Journal of Medical Sciences 29 730-734. (https:// doi.org/10.12669/pjms.293.3473)

Sonnylal S, Shi-Wen X, Leoni P, Naff K, Van Pelt CS, Nakamura H, Leask A, Abraham D, Bou-Gharios G \& de Crombrugghe B 2010 Selective expression of connective tissue growth factor in fibroblasts in vivo promotes systemic tissue fibrosis. Arthritis and Rheumatism 62 1523-1532. (https://doi.org/10.1002/art.27382)

Stanton H, Rogerson FM, East CJ, Golub SB, Lawlor KE, Meeker CT, Little CB, Last K, Farmer PJ, Campbell IK, et al. 2005 ADAMTS5 is the major aggrecanase in mouse cartilage in vivo and in vitro. Nature 434 648-652. (https://doi.org/10.1038/nature03417)

Tasharrofi B \& Ghafouri-Fard SL 2018 Long non-coding RNAs as regulators of the mitogen-activated protein kinase (MAPK) pathway in cancer. Klinicka Onkologie 31 95-102. (https://doi.org/10.14735/amko201895)
Thomson SE, McLennan SV, Hennessy A, Boughton P, Bonner J, Zoellner H, Yue DK \& Twigg SM 2010 A novel primate model of delayed wound healing in diabetes: dysregulation of connective tissue growth factor. Diabetologia 53 572-583. (https://doi. org/10.1007/s00125-009-1610-6)

Turns M 2012 The diabetic foot: an overview for community nurses. British Journal of Community Nursing 17 422, 424-427, 430-423. (https://doi.org/10.12968/bjcn.2012.17.9.422)

Wagner Jr FW 1981 The dysvascular foot: a system for diagnosis and treatment. Foot and Ankle 2 64-122. (https://doi. org/10.1177/107110078100200202)

Wang S, Li B, Li C, Cui W \& Miao L 2015 Potential renoprotective agents through inhibiting CTGF/CCN2 in diabetic nephropathy. Journal of Diabetes Research 2015 962383. (https://doi. org $/ 10.1155 / 2015 / 962383)$

Wang W, Yang C, Wang XY, Zhou LY, Lao GJ, Liu D, Wang C, Hu MD, Zeng TT, Yan L, et al. 2018 MicroRNA-129 and -335 promote diabetic wound healing by inhibiting Sp1-mediated MMP-9 expression. Diabetes 67 1627-1638. (https://doi.org/10.2337/db17-1238)

Yan B, Tao ZF, Li XM, Zhang H, Yao J \& Jiang Q 2014 Aberrant expression of long noncoding RNAs in early diabetic retinopathy. Investigative Ophthalmology and Visual Science 55 941-951. (https:// doi.org/10.1167/iovs.13-13221)

Yan W, Liu H, Deng X, Jin Y, Wang N \& Chu J 2018 Acellular dermal matrix scaffolds coated with connective tissue growth factor accelerate diabetic wound healing by increasing fibronectin through PKC signalling pathway. Journal of Tissue Engineering and Regenerative Medicine 12 e1461-e1473. (https://doi.org/10.1002/term.2564)

Yazdanpanah L, Nasiri M \& Adarvishi S 2015 Literature review on the management of diabetic foot ulcer. World Journal of Diabetes 6 37-53. (https://doi.org/10.4239/wjd.v6.i1.37)

Ye J, Kang Y, Sun X, Ni P, Wu M \& Lu S 2017 MicroRNA-155 inhibition promoted wound healing in diabetic rats. International Journal of Lower Extremity Wounds 16 74-84. (https://doi. org/10.1177/1534734617706636)

Yosimichi G, Nakanishi T, Nishida T, Hattori T, Takano-Yamamoto T \& Takigawa M 2001 CTGF/Hcs24 induces chondrocyte differentiation through a p38 mitogen-activated protein kinase (p38MAPK), and proliferation through a p44/42 MAPK/extracellular-signal regulated kinase (ERK). European Journal of Biochemistry 268 6058-6065. (https://doi.org/10.1046/j.0014-2956.2001.02553.x)

Zhang B, Zhou KK \& Ma JX 2010 Inhibition of connective tissue growth factor overexpression in diabetic retinopathy by SERPINA3K via blocking the WNT/beta-catenin pathway. Diabetes 59 1809-1816. (https://doi.org/10.2337/db09-1056)

Zhang J, Yang C, Wang C, Liu D, Lao G, Liang Y, Sun K, Luo H, Tan Q, Ren M, et al. 2016 AGE-induced keratinocyte MMP-9 expression is linked to TET2-mediated CpG demethylation. Wound Repair and Regeneration 24 489-500. (https://doi.org/10.1111/wrr.12426)

Zhang X, Hong R, Chen W, Xu M \& Wang L 2019 The role of long noncoding RNA in major human disease. Bioorganic Chemistry 92 103214. (https://doi.org/10.1016/j.bioorg.2019.103214)

Zhao M, Wang H, Chen J, Xi Y, Wang F, Huo C, Li W, Chu Y, Xu P, Huang Q et al. 2019 Expression of long non-coding RNA H19 in colorectal cancer patients with type 2 diabetes. Archives of Physiology and Biochemistry [epub]. (https://doi.org/10.1080/13813455.2019.1628068)

Zimprich A, Mroz G, Meyer Zu Reckendorf C, Anastasiadou S, Forstner P, Garrett L, Holter SM, Becker L, Rozman J, Prehn C, et al. 2017 Serum response factor (SRF) ablation interferes with acute stress-associated immediate and long-term coping mechanisms. Molecular Neurobiology 54 8242-8262. (https://doi.org/10.1007/s12035-016-0300-x)

Received in final form 14 April 2020

Accepted 13 July 2020

Accepted Manuscript published online 13 July 2020 https://jme.bioscientifica.com https://doi.org/10.1530/JME-19-0242 (c) 2020 Society for Endocrinology Published by Bioscientifica Ltd. Printed in Great Britain 